\title{
Three additive cubic equations
}

\author{
by
O. D. Atkinson (Sheffield), J. BrüDERn (Göttingen), and R. J. CoOK (Sheffield)

Introduction. The study of additive cubic diophantine equations falls naturally into two parts. The Hardy-Littlewood method establishes a Hasse principle, showing that if the equations have non-singular solutions in the $p$-adic fields then they have a non-trivial solution in rational integers. The second part of the problem is then to show the existence of $p$-adic solutions.

For a single equation,

$$
a_{1} x_{1}^{3}+\ldots+a_{N} x_{N}^{3}=0, \quad a_{i} \in \boldsymbol{Z},
$$

Baker [2] has applied Vaughan's improvements in the Hardy-Littlewood method to establish a Hasse principle provided that $N \geq 7$. The question of $p$-adic solutions was settled by Lewis [19] who showed that there are non-trivial $p$-adic solutions when $N \geq 7$. Further examples such as

$$
x_{1}^{3}-2 x_{2}^{3}+7\left(x_{3}^{3}-2 x_{4}^{3}\right)+49\left(x_{5}^{3}-2 x_{6}^{3}\right)=0
$$

show that the result does not hold when $N=6$. For a single equation, the non-trivial $p$-adic solutions are non-singular so we have

Proposition 1. The equation (0.1) has a non-trivial solution in integers provided that $N \geq 7$.

Davenport and Lewis [15] considered pairs of additive cubic equations

$$
\begin{aligned}
& a_{1} x_{1}^{3}+\ldots+a_{N} x_{N}^{3}=0, \\
& b_{1} x_{1}^{3}+\ldots+b_{N} x_{N}^{3}=0
\end{aligned}
$$

with integer coefficients $a_{i}, b_{i}$. They established the Hasse principle if $N \geq 18$, subject to a rank condition on the coefficients. This was reduced successively by Cook [11], Vaughan [22], Baker and Brüdern [3] and Brüdern [6], it now holds for $N \geq 14$. Davenport and Lewis also showed that the equations (0.3) have non-trivial $p$-adic solutions when $N \geq 16$, and examples 
such as

$$
\begin{gathered}
\Phi\left(x_{1}, \ldots, x_{5}\right)+7 \Phi\left(y_{1}, \ldots, y_{5}\right)+49 \Phi\left(z_{1}, \ldots, z_{5}\right)=0 \\
\Psi\left(x_{1}, \ldots, x_{5}\right)+7 \Psi\left(y_{1}, \ldots, y_{5}\right)+49 \Psi\left(z_{1}, \ldots, z_{5}\right)=0,
\end{gathered}
$$

where

$$
\begin{aligned}
& \Phi\left(x_{1}, \ldots, x_{5}\right)=x_{1}^{3}+2 x_{2}^{3}+6 x_{3}^{3}-4 x_{4}^{3}, \\
& \Psi\left(x_{1}, \ldots, x_{5}\right)=x_{2}^{3}+2 x_{3}^{3}+4 x_{4}^{3}+x_{5}^{3}
\end{aligned}
$$

have no non-trivial solution in the 7-adic field. Cook [12] showed that if $p \neq 7$ then the equations (0.3) have a non-trivial $p$-adic solution if $N \geq 13$.

In the case of two equations (0.3), non-trivial $p$-adic solutions are not necessarily non-singular. However, Davenport and Lewis [15], Corollary to Theorem 1, proved that the existence of non-trivial $p$-adic solutions implies the existence of a non-singular $p$-adic solution provided that the columns of coefficients satisfy a rank condition, namely that no ratio occurs more than six times among the $a_{i} / b_{i}$. When this rank condition holds they established the Hasse principle via the analytic arguments of the Hardy-Littlewood method. When it does not hold, Proposition 1 combined with elementary arguments establishes the existence of non-trivial integer solutions to the equations (0.3). We have

Proposition 2. The equations (0.3) have a non-trivial solution in integers provided that $N \geq 16$.

Here we are concerned with three simultaneous additive cubic equations

$$
\begin{aligned}
& a_{1} x_{1}^{3}+\ldots+a_{N} x_{N}^{3}=0, \\
& b_{1} x_{1}^{3}+\ldots+b_{N} x_{N}^{3}=0, \\
& c_{1} x_{1}^{3}+\ldots+c_{N} x_{N}^{3}=0
\end{aligned}
$$

with integer coefficients. Little has been published explicitly on this problem, although some information can be obtained from more general results. For example, early results of Brauer [5] and Birch [4] would need a large number of variables. Davenport and Lewis [17] considered systems of additive diophantine equations

$$
a_{i 1} x_{1}^{k}+\ldots+a_{i N} x_{i N}^{k}=0, \quad i=1, \ldots, R
$$

with integer coefficients. They found that the system (0.7) always has nontrivial solutions, both in integers and in the $p$-adic fields, provided that

$$
N \geq \begin{cases}{\left[9 R^{2} k \log k\right]} & \text { for } k \text { odd } \\ {\left[48 R^{2} k^{3} \log 3 R k^{2}\right]} & \text { for } k \text { even, } k>2 .\end{cases}
$$

Clearly one would hope to do better for particular small values of $R$ and $k$. In the case of the equations (0.6) one can apply a variant of Hua's Lemma (see Cook [10]) to establish the Hasse principle when $N \geq 25$, provided that 
the columns of coefficients satisfy a rank condition. Recent improvements in the Hardy-Littlewood method enable us to do better than this.

As with pairs of equations, there are combinatorial problems arising from linear dependence amongst the columns in the matrix of coefficients of the system. We shall call an $r \times n r$ matrix partitionable if it consists of $n$ disjoint $r \times r$ submatrices which all have non-zero determinant. In the case $r=2$ the situation is fairly simple; a submatrix $\left[\begin{array}{cc}a_{i} & a_{j} \\ b_{i} & b_{j}\end{array}\right]$ has non-zero determinant if and only if the ratios $a_{i} / b_{i}$ and $a_{j} / b_{j}$ are distinct. Therefore, in this case, whether a matrix is partitionable or not, can easily be seen from repetitions in the sequence $a_{i} / b_{i}$.

However, when $r \geq 3$ things become much more difficult, a fact which might have obstructed further progress with problems of this class. Low, Pitman and Wolff [20] recognized that the appropriate analogue to control partitionable matrices is the following combinatorial result (for a proof see Aigner [1] or Low, Pitman and Wolff [20]).

Proposition 3. An $r \times r n$ matrix $A$ over a field is partitionable if and only if every submatrix consisting of $t$ columns from $A$ has rank $\geq t / n$.

There will be many delicate questions associated with relatively large submatrices of low rank. We shall establish the Hasse principle for the system (0.6), via the Hardy-Littlewood method, subject to a rank condition on the matrix of coefficients. If the system does not satisfy this rank condition then it is easy to establish the existence of non-trivial integer solutions from Propositions 1 and 2.

Definition. We say that the system (0.6) satisfies the rank condition if no six columns of coefficients form a matrix of rank 1 and no fifteen columns of coefficients form a matrix of rank 2 .

Theorem 1 (Hasse principle). Suppose that in the system (0.6), $N \geq$ 22 , the rank condition is satisfied and that there is a non-singular solution in every p-adic field. Then the system (0.6) has a non-trivial solution in rational integers.

The rank condition, and the condition $N \geq 22$, allow us to apply the Hardy-Littlewood method to the problem. We obtain an asymptotic formula for the number of solutions to (0.6) in a 22-dimensional box of side $(C-1) P$, where $C>1$ is some suitable constant, with some additional restrictions on the variables. The main term is

$$
\Gamma \mathfrak{S} P^{13}
$$

where $\Gamma$ is a positive constant and $\mathfrak{S}$ is the singular series. Then $\mathfrak{S}$ is bounded below by some positive constant if and only if there is a non- 
singular solution to the system (0.6) in every $p$-adic field.

We may take $N=22$, by setting excess variables to the value 0 . The rank condition is then equivalent to the conditions that

(i) any non-trivial linear combination of the equations (0.6) contains at least 7 variables explicitly;

(ii) any two linearly independent linear combinations of the equations (0.6) contain at least 16 variables explicitly.

It is now straightforward to modify the arguments of $\S 5$ of Davenport and Lewis [17] to obtain the following.

Proposition 4. Suppose that in the system (0.6), $N \geq 22$ and the rank condition is satisfied. For any prime $p$, if the system has a non-trivial solution in the p-adic field then it has a non-singular solution in the p-adic field.

We may now replace Theorem 1 with

Theorem 1A. Suppose that in the system (0.6), $N \geq 22$, the rank condition is satisfied and that there is a non-trivial solution in every $p$-adic field. Then the system (0.6) has a non-trivial solution in rational integers.

Thus the problem now reduces to establishing that the equations (0.6) have non-trivial $p$-adic solutions. The bounds (0.8) have been reduced by Cook [13] and Low, Pitman and Wolff [20]. In the particular case (0.6) of three additive cubic equations Stevenson [21] showed that if $N \geq 28$ then the equations have non-trivial $p$-adic solutions except possibly when $p=3,7$.

Theorem 2. Suppose that $N \geq 22$. Then the equations (0.6) have nontrivial p-adic solutions except (possibly) for $p=3$ and for those primes $p \equiv 1 \bmod 3$ with $p \leq 43$.

Combining Theorems $1 \mathrm{~A}$ and 2 with elementary arguments when the rank condition fails we obtain

TheOREM 3. Suppose that the equations (0.6) have $N \geq 22$ and a nontrivial $p$-adic solution for $p=3$ and for those primes $p \equiv 1 \bmod 3$ with $p \leq 43$. Then the equations (0.6) have a non-trivial solution in rational integers.

For any given set of equations (0.6) it is easy to check whether the $p$ adic conditions are satisfied using a computer. The number of variables is essentially best possible since combining the counter-examples $(0.2)$ and (0.4) gives a system of three equations in 21 variables having no non-trivial 7 -adic solution. Further, if we adjoin a third congruence, say

$$
\Xi=x_{6}^{3}+x_{7}^{3}+x_{8}^{3} \equiv 0 \bmod 7,
$$


to $\Phi$ and $\Psi$ in (0.5) we obtain a system of three congruences modulo 7 which only have singular solutions. This leads to a system of three equations in 24 variables for which we cannot use Hensel's Lemma to establish the existence of 7 -adic solutions.

Thus any refinement of the Hardy-Littlewood method to deal with three cubic forms in 21 (or fewer) variables must impose conditions on 7-adic solutions. It seems likely that the arguments would be much more involved (see [2] and [6] for the corresponding results for one or two cubic forms). Further, an analogue of Theorem 2 when $N=21$ would lead to a larger set of exceptional primes.

\section{CHAPTER 1}

\section{THE HASSE PRINCIPLE}

1. Excluding simple cases. There are some situations in which it is very easy to find solutions to the system (0.6). In order to formulate this in a suitable and brief manner we introduce some notation. Let

$$
M=\left[\begin{array}{lll}
a_{1} & \ldots & a_{22} \\
b_{1} & \ldots & b_{22} \\
c_{1} & \ldots & c_{22}
\end{array}\right]
$$

be the matrix of coefficients associated to (0.6). Also, for any subset $\mathcal{J} \subset$ $\{1,2, \ldots, 22\}$, we let

$$
M_{\mathcal{J}}=\left[\begin{array}{c}
a_{j} \\
b_{j} \\
c_{j}
\end{array}\right]_{j \in \mathcal{J}}
$$

be the submatrix of $M$ consisting of the $j$ th columns, $j \in \mathcal{J}$.

In the arguments below we shall often "normalize" the system (0.6), that is, taking linear combinations of the three equations, in order to produce, for example, a large number of zeros in a row. Also we shall re-index the variables to collect such zeros together, into blocks. We shall then always redenote the coefficients by $a_{i}, b_{i}, c_{i}$ without further comments. This should not lead to confusion, and avoids abundant notational complications.

Of course we may assume at once that any column contains at least one non-zero coefficient; otherwise there is a non-trivial solution to (0.6) for obvious reasons.

Lemma 1. Suppose there is a $\mathcal{J} \subset\{1, \ldots, 22\}$ with $|\mathcal{J}| \geq 6$ and $\operatorname{rk}\left(M_{\mathcal{J}}\right)$ $=1$. Then (0.6) has a non-trivial integer solution.

Proof. Suppose $|\mathcal{J}|=6$ and $\operatorname{rk}\left(M_{\mathcal{J}}\right)=1$. Normalizing the system (0.6) we may assume that $b_{i}=c_{i}=0$ for $1 \leq i \leq 6$. By Proposition 2 there 
is a $\boldsymbol{y}=\left(y_{7}, \ldots, y_{22}\right) \neq 0$ with

$$
\begin{aligned}
& b_{7} y_{7}^{3}+\ldots+b_{22} y_{22}^{3}=0, \\
& c_{7} y_{7}^{3}+\ldots+c_{22} y_{22}^{3}=0 .
\end{aligned}
$$

But if $\boldsymbol{y}$ is a solution, then $\eta \boldsymbol{y}$ also solves (1.1) for all $\eta \in \boldsymbol{Z}$. Putting these solutions into the remaining equation we are left with the single equation

$$
a_{1} x_{1}^{3}+\ldots+a_{6} x_{6}^{3}+a(\boldsymbol{y}) \eta^{3}=0
$$

for some integer $a(\boldsymbol{y})$. This has an integer solution $\left(x_{1}, \ldots, x_{6}, \eta\right)$ by Proposition 1. Now $\left(x_{1}, \ldots, x_{6}, \eta y_{7}, \ldots, \eta y_{22}\right)$ solves $(0.6)$.

Lemma 2. Suppose there is a $\mathcal{J} \subset\{1, \ldots, 22\}$ with $|\mathcal{J}| \geq 15$ and $\operatorname{rk}\left(M_{\mathcal{J}}\right)=2$. Then (0.6) has a non-trivial integer solution.

Pr o of. Here we may assume that $c_{1}=\ldots=c_{15}=0$. Thus, solving the single equation $c_{16} x_{16}^{3}+\ldots+c_{22} x_{22}^{3}=0$ first, and then using an argument similar to the one just given, one is left with a pair of equations in 16 variables to which Proposition 2 may again be applied.

By Lemmata 1 and 2, if (0.6) does not satisfy the rank condition, then (0.6) has a non-trivial integer solution. In other words, Theorem 3 follows from Theorems 1A and 2. Hence, for the remainder of this chapter we shall assume that the rank condition is satisfied.

Based on this assumption we are now able to deduce the following result.

LEMMA 3. There is a set $\mathcal{S} \subset\{1, \ldots, 22\}$ with $|\mathcal{S}|=4$ such that for all $\mathcal{T} \subset \mathcal{S}$ with $|\mathcal{T}|=3$ one has $\operatorname{det} M_{\mathcal{T}} \neq 0$.

Proof. Since $M$ has rank 3 , there is a $3 \times 3$ submatrix, $M_{1,2,3}$ say, with non-zero determinant. Taking linear combinations we may suppose that $a_{2}=a_{3}=b_{1}=b_{3}=c_{1}=c_{2}=0$. Now, if there is a column, the $j$ th, say, with all entries non-zero, then $|\mathcal{S}|$ has the required properties. If there is no such column then any column contains at least one zero, and after redenoting the coefficients the matrix $M$ must be of the shape

$$
M=\left[\begin{array}{cccccc}
a_{1}^{\prime} \ldots a_{u}^{\prime} & 0 \ldots 0 & 0 \ldots 0 & a_{1}^{\prime \prime} \ldots a_{l}^{\prime \prime} & a_{1}^{\prime \prime \prime} \ldots a_{m}^{\prime \prime \prime} & 0 \ldots 0 \\
0 \ldots 0 & b_{1}^{\prime} \ldots b_{v}^{\prime} & 0 \ldots 0 & b_{1}^{\prime \prime} \ldots b_{l}^{\prime \prime} & 0 \ldots 0 & b_{1}^{\prime \prime \prime} \ldots b_{n}^{\prime \prime \prime \prime} \\
0 \ldots 0 & 0 \ldots 0 & c_{1}^{\prime} \ldots c_{w}^{\prime} & 0 \ldots 0 & c_{1}^{\prime \prime \prime} \ldots c_{m}^{\prime \prime \prime} & c_{1}^{\prime \prime \prime \prime} \ldots c_{n}^{\prime \prime \prime}
\end{array}\right]
$$

with $u+v+w+l+m+n=22$, with all entries occurring explicitly being nonzero, and with $a_{1}^{\prime}=a_{1}, b_{1}^{\prime}=b_{2}, c_{1}^{\prime}=c_{3}$. By our conditions on submatrices of rank 1 and 2 we also have

$$
\begin{gathered}
u \leq 5, \quad v \leq 5, \quad w \leq 5, \\
u+v+l \leq 14, \quad u+w+m \leq 14, \quad v+w+n \leq 14 .
\end{gathered}
$$


We now easily deduce that at least two of the numbers $l, m, n$ are non-zero. Without lost of generality we may suppose that $m n \neq 0$. Then the matrix

$$
\left[\begin{array}{cccc}
a_{1}^{\prime} & 0 & a_{1}^{\prime \prime \prime} & 0 \\
0 & b_{1}^{\prime} & 0 & b_{1}^{\prime \prime \prime \prime} \\
0 & 0 & c_{1}^{\prime \prime \prime} & c_{1}^{\prime \prime \prime \prime}
\end{array}\right]
$$

has the required properties.

2. Preparing the equations for the circle method. The treatment of the system (0.6) now depends on whether $M$ contains a large submatrix of rank 2, or not. Let $R$ be the maximal number of columns of $M$ forming an $3 \times R$ matrix of rank 2 . Of course $R \leq 14$.

Case I. $R \leq 12$. In this case we first pick four indices according to Lemma 3. We may suppose that these are 1, 2, 3, 4. Taking linear combinations we may also suppose that $b_{1}=c_{1}=a_{2}=c_{2}=a_{3}=b_{3}=0$. A prominent role will be played by the ternary linear forms

$$
\gamma_{i}=a_{i} \alpha_{1}+b_{i} \alpha_{2}+c_{i} \alpha_{3} \quad(1 \leq i \leq 22) .
$$

Let $\{j, k, l\} \subset\{1,2,3,4\}$. Then $\operatorname{det} M_{j, k, l} \neq 0$. Therefore, for all $i \geq 5$ we may write

$$
\gamma_{i}=\vartheta \gamma_{j}+\lambda \gamma_{k}+\mu \gamma_{l}
$$

where $\vartheta, \lambda, \mu$ are suitable rational numbers depending on $i, j, k, l$. However, if in (0.6) the variables $x_{v}$, for $v \geq 5$, are replaced by $d_{v} x_{v}$ with suitable integers $d_{v} \neq 0$, then we may assume that $\vartheta, \lambda, \mu$ are always integers, as we shall from now on suppose.

Case II. $13 \leq R \leq 14$. Here we choose a maximal submatrix, of $R$ columns and of rank 2 . We may suppose that this is $M_{\mathcal{K}}$ where $\mathcal{K}=$ $\{1,2,25-R, 26-R, \ldots, 22\}$. Taking linear combinations we arrange that

$$
M=\left[\begin{array}{cccccccccc}
a_{1} & 0 & 0 & a_{4} & \ldots & a_{10} & a_{11} & a_{12} & \ldots & a_{22} \\
0 & b_{2} & 0 & b_{4} & \ldots & b_{10} & b_{11} & b_{12} & \ldots & b_{22} \\
0 & 0 & c_{3} & c_{4} & \ldots & c_{10} & c_{11} & 0 & \ldots & 0
\end{array}\right]
$$

where $c_{i} \neq 0$ for $3 \leq i \leq 10$, and $c_{11}=0$ or $c_{11} \neq 0$ depending on whether $R=14$ or $R=13$.

In this case Lemma 3 is not very useful. A partial surrogate can be obtained as follows. The numbers $a_{4}, \ldots, a_{10}, b_{4}, \ldots, b_{10}$ cannot all be zero since this would imply that there is a $3 \times 6$ submatrix of rank 1 . As we may exchange the rows with the $a_{i}$ 's and the $b_{i}$ 's by symmetry we may assume that $a_{4} \neq 0$. Hence at least the matrices $M_{1,2,3}, M_{1,2,4}$, and $M_{2,3,4}$ have non-zero determinant. Hence we have (1.3) with $i, j, k$ any of these triples, and as in Case I we suppose that $\vartheta, \lambda, \mu$ in (1.3) are integers. 
One final normalization of the system (0.6) is now required which applies to both cases. Since (0.6) is a system of odd degree there are of course nonsingular real solutions $\boldsymbol{\xi}=\left(\xi_{1}, \ldots, \xi_{22}\right)$ to $(0.6)$ with all $\xi_{i} \neq 0$. By changing certain variables $x_{i}$ into $-x_{i}$ if necessary we may also suppose $\xi_{i}>0$; and since (0.6) is homogeneous even $\xi_{i}>1$ may be assumed. For later use we now fix a constant $C$ with

$$
\xi_{i}^{3} \leq C \quad(1 \leq i \leq 22) .
$$

All these conventions understood, we are now prepared to apply a threedimensional version of the Hardy-Littlewood method. Let $P$ be a large parameter tending to infinity, and let

$$
\mathcal{A}=\left\{P<x \leq C P: p \mid x \Rightarrow p \leq P^{\eta}\right\}
$$

where $\eta$ is a small positive constant to be fixed later on. Let $\mathcal{N}$ be the number of solutions of (0.6) subject to

$$
\begin{aligned}
P<x_{i} \leq C P & (1 \leq i \leq 4), \\
x_{i} \in \mathcal{A} & (5 \leq i \leq 22) .
\end{aligned}
$$

Bringing in the exponential sums

$$
\begin{aligned}
& f(\alpha)=\sum_{P<x \leq C P} e\left(\alpha x^{3}\right), \\
& g(\alpha)=\sum_{x \in \mathcal{A}} e\left(\alpha x^{3}\right),
\end{aligned}
$$

and recalling (1.2), we may write

$$
\mathcal{N}=\int_{\mathfrak{U}} f\left(\gamma_{1}\right) f\left(\gamma_{2}\right) f\left(\gamma_{3}\right) G(\boldsymbol{\alpha}) d \boldsymbol{\alpha}
$$

where $\mathfrak{U}$ is the three-dimensional unit cube $\left[(\log P) P^{-3}, 1+(\log P) P^{-3}\right]^{3}$, and

$$
G(\boldsymbol{\alpha})=\prod_{i=5}^{22} g\left(\gamma_{i}\right) .
$$

The unit cube is dissected into major and minor "arcs" as follows. Let $\delta>0$ be a fixed small real number with $4 \eta<\delta$, and let $\mathfrak{M}(q, \boldsymbol{t})$ denote the box

$$
\left\{\boldsymbol{\alpha}:\left|\alpha_{i}-t_{i} / q\right| \leq(\log P)^{\delta} P^{-3}\right\},
$$

and write $\mathfrak{M}$ for the union of all $\mathfrak{M}(q, \boldsymbol{t})$ with $1 \leq t_{i} \leq q \leq(\log P)^{\delta}$ for $1 \leq i \leq 3$, and $\left(q, t_{1}, t_{2}, t_{3}\right)=1$. Let $\mathfrak{m}=\mathfrak{U} \backslash \mathfrak{M}$.

If $\mathfrak{B} \subset \mathfrak{U}$ is a measurable set it is useful to write $\mathcal{N}(\mathfrak{B})$ for the integral (1.10) with integration restricted to the subset $\mathfrak{B}$. 
In $\S \S 4$ and 5 we shall show that

$$
\mathcal{N}(\mathfrak{m})=o\left(P^{13}\right),
$$

by arguments which are different in the two cases. In $\S 6$ the major arcs are treated, and we will find an asymptotic formula

$$
\mathcal{N}(\mathfrak{M})=\Gamma \mathfrak{S} P^{13}+o\left(P^{13}\right)
$$

where $\Gamma$ is a certain positive constant, and $\mathfrak{S}$ is the singular series defined in (1.67) below. Finally, the singular series will be related to the $p$-adic solubility of (0.6). In the next section, however, we collect various auxiliary results for frequent use later on.

3. Auxiliary estimates. We begin with various well known properties of the exponential sum $f(\alpha)$. It is convenient to introduce the intervals

$$
\mathfrak{E}(q, t)=\left\{\alpha:|q \alpha-t| \leq P^{-9 / 4}\right\}
$$

and to write $\mathfrak{E}$ for the union of all $\mathfrak{E}(q, t)$ with $1 \leq t \leq q \leq P^{3 / 4},(t, q)=1$.

Lemma 4. Let $0<\delta<1 / 4$. Then either $|f(\alpha)|<P^{3 / 4+\delta}$, or $\alpha(\bmod 1)$ $\in \mathfrak{E}$.

This is a slightly amplified form of Weyl's inequality; see Vaughan [24], Lemma 1.

For our next results we require the functions

$$
\begin{aligned}
S(q, t) & =\sum_{x=1}^{q} e\left(\frac{t x^{3}}{q}\right) ; \\
w(\beta) & =\int_{P}^{C P} e\left(\beta \gamma^{3}\right) d \gamma .
\end{aligned}
$$

We also introduce a multiplicative function $\kappa(q)$, defined at prime powers by

$$
\kappa\left(p^{3 l+1}\right)=2 p^{-l-1 / 2} ; \quad \kappa\left(p^{3 l+2}\right)=p^{-l-1} ; \quad \kappa\left(p^{3 l}\right)=p^{-l},
$$

and remark that by Lemmata 4.3 and 4.4 of Vaughan [23] we have

$$
q^{-1} S(q, t) \ll \kappa(q)
$$

whenever $(q, t)=1$.

Lemma 5. For $\alpha \in \mathfrak{E}(q, t)$,

$$
f(\alpha)=q^{-1} S(q, t) w(\alpha-t / q)+O\left(q^{1 / 2+\varepsilon}\right) .
$$

When $(q, t)=1$ and $q \leq P^{3 / 4}$ we also have

$$
f(\alpha) \ll P \kappa(q)\left(1+P^{3}|\alpha-t / q|\right)^{-1 / 2} .
$$


Proof. The first part is Theorem 4.1 of Vaughan [23]. The second part is easily deduced therefrom by using $\kappa(q) \geq q^{-1 / 2}$ and an estimate for $w(\beta)$ obtained from a partial integration in (1.16).

From the definition of $\kappa$ we have

$$
\sum_{q \leq X} q \kappa(q)^{4} \leq \prod_{p \leq X}\left(1+\sum_{l=1}^{\infty} p^{l} \kappa\left(p^{l}\right)^{4}\right) \ll \prod_{p \leq X}\left(1+\frac{10}{p}\right) \ll(\log X)^{10} .
$$

Now the following estimates are immediate.

Lemma 6. We have

$$
\begin{gathered}
\int_{\mathfrak{E}}|f(\alpha)|^{4} d \alpha \ll P(\log P)^{10}, \\
\int_{\mathfrak{E}}|f(\alpha)|^{5} d \alpha \ll P^{2} .
\end{gathered}
$$

We also need mean value estimates of Hua's Lemma type. We collect these in our next lemma.

Lemma 7. Let $s \geq 4$. Then

$$
\int_{0}^{1}|g(\alpha)|^{s} d \alpha \ll P^{\vartheta(s)}
$$

where

$$
\begin{gathered}
\vartheta(4)=2+\varepsilon, \quad \vartheta(6)=13 / 4+\varepsilon, \quad \vartheta(20 / 3)=23 / 6+\varepsilon, \\
\vartheta(7)=33 / 8+\varepsilon, \quad \vartheta(8)=5 .
\end{gathered}
$$

Proof. The value for $\vartheta(6)$ follows from Theorem 4.4 of Vaughan [25] providing $\eta$ is sufficiently small. Interpreting the integral as the number of solutions of the underlying diophantine equation when $s=4$ or 8 , the values for $\vartheta(4)$ and $\vartheta(8)$ follow from Hua's Lemma (Vaughan [23], Lemma 2.5) and Theorem 2 of Vaughan [24]. The other values now can be deduced via Hölder's inequality.

Lemma 8. Let $\nu \neq 0$ be fixed. Then, uniformly in $\mu \in \boldsymbol{R}$,

$$
\int_{0}^{1}\left|f(\alpha) g(\nu \alpha+\mu)^{6}\right| d \alpha \ll P^{4+\varepsilon} .
$$

Proof. Let $\delta>0$ be small. Since $g(\alpha)$ has period one,

$$
\int_{0}^{1}|g(\nu \alpha+\mu)|^{6} d \alpha \leq \frac{|\nu|+1}{|\nu|} \int_{0}^{1}|g(\beta)|^{6} d \beta \ll P^{13 / 4+\varepsilon}
$$


by Lemma 7. Therefore the contribution from all $\alpha \in[0,1]$ where $|f(\alpha)|<$ $P^{3 / 4+\delta}$ is $\ll P^{4+2 \delta}$. For the remaining $\alpha$ we have $\alpha(\bmod 1) \in \mathfrak{E}$. By Hölder's inequality and Lemmata 6 and 7 ,

$$
\begin{aligned}
\int_{\mathfrak{E}} \mid f(\alpha) g(\nu \alpha & +\mu)^{6} \mid d \alpha \\
\leq & \left(\int_{\mathfrak{E}}|f(\alpha)|^{4} d \alpha\right)^{1 / 4}\left(\int_{0}^{1}|g(\nu \alpha+\mu)|^{8} d \alpha\right)^{3 / 4} \ll P^{4+\varepsilon} .
\end{aligned}
$$

As $\delta$ was arbitrarily small this proves the lemma.

The next two lemmata are variants of Lemmata 2.1 and 2.2 of Brüdern [6], which are more convenient in the present context.

Lemma 9. Let $\Psi(\alpha)$ be a trigonometric sum,

$$
\Psi(\alpha)=\sum_{|h| \leq H} \psi(h) e(\alpha h)
$$

where $\log H \ll \log P$, and where $\Psi(\alpha) \geq 0$ for all $\alpha \in \boldsymbol{R}$. Then

$$
\int_{\mathfrak{E}}|f(\alpha)|^{3} \Psi(\alpha) d \alpha \ll P^{3 / 8+\varepsilon} \psi(0)+P^{\varepsilon} \sum_{h \neq 0}|\psi(h)| .
$$

Lemma 10. Let $\Psi(\alpha, \beta)$ be a double trigonometric sum,

$$
\Psi(\alpha, \beta)=\sum_{|h|,|k| \leq H} \psi(h) e(\alpha h+\beta k)
$$

where $\log H \ll \log P$, and where $\Psi(\alpha, \beta) \geq 0$ for all $(\alpha, \beta) \in \boldsymbol{R}^{2}$. Then

$$
\begin{aligned}
& \int_{\mathfrak{E}} \int_{\mathfrak{E}}|f(\alpha)|^{3}|f(\beta)|^{3} \Psi(\alpha, \beta) d \alpha \\
& \ll P^{3 / 4+\varepsilon} \psi(0,0)+P^{3 / 8+\varepsilon} \sum_{h \neq 0}(|\psi(h, 0)|+|\psi(0, h)|)+P^{\varepsilon} \sum_{h, k}|\psi(h, k)| .
\end{aligned}
$$

We only sketch a proof of Lemma 10. Lemma 9 can be established by the same method. Using Lemma 5 and the argument in $\S 2$ of Brüdern [6] one readily establishes that the left hand side of the proposed inequality in Lemma 10 is

$$
\ll P^{\varepsilon} \sum_{q \leq P^{3 / 4}} \sum_{r \leq P^{3 / 4}} \kappa(q)^{3} \kappa(r)^{3} \sum_{h, k}\left|\psi(h, k) \| c_{q}(h) c_{r}(k)\right|
$$

where $c_{q}(h)$ is Ramanujan's sum,

$$
c_{q}(h)=\sum_{\substack{a=1 \\(a, q)=1}}^{q} e(a h / q) .
$$


One now observes that $\kappa(q) \ll q^{\varepsilon-1 / 3}$, and that

$$
\sum_{q \leq X} q \kappa(q)^{3} \ll X^{1 / 2+\varepsilon} .
$$

The proof can now be completed as in $\S 2$ of [6].

4. The minor arcs in Case I. In this section it is assumed throughout that $M$ is of the special shape specified in $\S 2$. We first draw a number of corollaries from the results of the previous section. They all rely on the trivial inequality

$$
\left|z_{1} \ldots z_{m}\right| \leq\left|z_{1}\right|^{m}+\ldots+\left|z_{m}\right|^{m} .
$$

Let $5 \leq i<j<k \leq 22$, and suppose that $\operatorname{det} M_{i, j, k} \neq 0$. Then the change of variable $\left(\alpha_{1}, \alpha_{2}, \alpha_{3}\right) \rightarrow\left(\gamma_{i}, \gamma_{j}, \gamma_{k}\right)$ is non-singular. Therefore, by (1.2) and Lemma 7,

$$
\begin{aligned}
\int_{\mathfrak{U}}\left|g\left(\gamma_{i}\right) g\left(\gamma_{j}\right) g\left(\gamma_{k}\right)\right|^{6} d \boldsymbol{\alpha} \\
\\
\ll \int_{0}^{1} \int_{0}^{1} \int_{0}^{1}\left|g\left(\gamma_{i}\right) g\left(\gamma_{j}\right) g\left(\gamma_{k}\right)\right|^{6} d \gamma_{i} d \gamma_{j} d \gamma_{k} \ll P^{39 / 4+\varepsilon} .
\end{aligned}
$$

In the matrix $M_{5,6, \ldots, 22}$ there are no six columns forming a $3 \times 6$ submatrix of rank 1, and no 13 columns forming a submatrix of rank 2. By Proposition 2, $M_{5,6, \ldots, 22}$ is partitionable. Therefore we may apply (1.18) to deduce from (1.11) that

$$
G(\boldsymbol{\alpha}) \leq \sum_{i, j, k}^{\prime}\left|g\left(\gamma_{i}\right) g\left(\gamma_{j}\right) g\left(\gamma_{k}\right)\right|^{6}
$$

where $\sum^{\prime}$ denotes a sum over all $i, j, k$ with $5 \leq i<j<k \leq 22$ and $\operatorname{det} M_{i, j, k} \neq 0$. Integrating this over $\mathfrak{U}$ we obtain

$$
\int_{\mathfrak{U}}|G(\boldsymbol{\alpha})| d \boldsymbol{\alpha} \ll P^{39 / 4+\varepsilon} .
$$

The same argument, but using the case $s=8$ of Lemma 7, gives

$$
\int_{\mathfrak{U}}|G(\boldsymbol{\alpha})|^{4 / 3} d \boldsymbol{\alpha} \ll P^{15} .
$$

Let $\{i, j, k\} \subset\{1,2,3,4\}$. Again we have that $\operatorname{det} M_{i, j, k} \neq 0$. Denote the set of all $\boldsymbol{\alpha} \in \mathfrak{U}$ where $\left|f\left(\gamma_{l}\right)\right|>P^{3 / 4+\delta}$ for all $l \in\{i, j, k\}$, by $\mathfrak{B}(i, j, k)$. 
Then $\gamma_{l} \in \mathfrak{E}$, and from Lemma 6 we find that

$$
\begin{aligned}
\int_{\mathfrak{B}(i, j, k)}\left|f\left(\gamma_{i}\right) f\left(\gamma_{j}\right) f\left(\gamma_{k}\right)\right|^{4} d \boldsymbol{\alpha} \\
\qquad \int_{\mathfrak{E}} \int_{\mathfrak{E}} \int_{\mathfrak{E}}\left|f\left(\gamma_{i}\right) f\left(\gamma_{j}\right) f\left(\gamma_{k}\right)\right|^{4} d \gamma_{i} d \gamma_{j} d \gamma_{k} \ll P^{3+\varepsilon} .
\end{aligned}
$$

We dissect the minor arcs further, according to the size of the $\left|f\left(\gamma_{l}\right)\right|$. The procedure is not dissimilar from our recent work on cubic diophantine inequalities [7]. Let $i, j, k, l$ be any permutation of $1,2,3,4$, and put

$$
\begin{aligned}
\mathfrak{F} & =\left\{\boldsymbol{\alpha} \in \mathfrak{m}:\left|f\left(\gamma_{v}\right)\right|<P^{3 / 4+\delta}(1 \leq v \leq 4)\right\}, \\
\mathfrak{G}_{i} & =\left\{\boldsymbol{\alpha} \in \mathfrak{m}:\left|f\left(\gamma_{i}\right)\right| \geq P^{3 / 4+\delta} ;\left|f\left(\gamma_{v}\right)\right|<P^{3 / 4+\delta}(1 \leq v \leq 4 ; v \neq i)\right\}, \\
\mathfrak{H}_{i j} & =\left\{\boldsymbol{\alpha} \in \mathfrak{m}:\left|f\left(\gamma_{v}\right)\right| \geq P^{3 / 4+\delta}(v=i, j) ;\left|f\left(\gamma_{w}\right)\right|<P^{3 / 4+\delta}(w=k, l)\right\}, \\
\mathfrak{K}_{i} & =\left\{\boldsymbol{\alpha} \in \mathfrak{m}:\left|f\left(\gamma_{i}\right)\right|<P^{3 / 4+\delta} ;\left|f\left(\gamma_{v}\right)\right| \geq P^{3 / 4+\delta}(1 \leq v \leq 4 ; v \neq i)\right\}, \\
\mathfrak{L} & =\left\{\boldsymbol{\alpha} \in \mathfrak{m}:\left|f\left(\gamma_{v}\right)\right| \geq P^{3 / 4+\delta}(1 \leq v \leq 4)\right\} .
\end{aligned}
$$

It is clear that any $\boldsymbol{\alpha} \in \mathfrak{m}$ is contained in one of these sets. If $\mathfrak{B}$ denotes any of these sets we have to show that $\mathcal{N}(\mathfrak{B})=o\left(P^{13}\right)$. From the definition of $\mathcal{N}(\mathfrak{B})$ and (1.19) we immediately have

$$
\mathcal{N}(\mathfrak{F}) \ll P^{3+4 \delta+39 / 4+\varepsilon} \ll P^{13-\delta} .
$$

The set $\mathfrak{K}_{i}$ is also fairly simple. Note that $\boldsymbol{\alpha} \in \mathfrak{K}_{i}$ implies $\boldsymbol{\alpha} \in \mathfrak{B}(j, k, l)$. Hence, by (1.20), (1.21) and Hölder's inequality,

$$
\mathcal{N}\left(\mathfrak{K}_{i}\right) \ll P^{3 / 4+\delta}\left(P^{3+\varepsilon}\right)^{1 / 4}\left(P^{15}\right)^{3 / 4} \ll P^{13-\delta} .
$$

For the set $\mathfrak{L}$ we also wish to use (1.20) and Hölder's inequality. Then $\mathcal{N}(\mathfrak{L})=o\left(P^{13}\right)$ would follow providing we can show that

$$
\int_{\mathfrak{L}}\left|f\left(\gamma_{1}\right) f\left(\gamma_{2}\right) f\left(\gamma_{3}\right) f\left(\gamma_{4}\right)\right|^{4} d \boldsymbol{\alpha}=o\left(P^{7}\right)
$$

To prove this first note that $\boldsymbol{\alpha} \in \mathfrak{L}$ implies $\boldsymbol{\alpha} \in \mathfrak{B}(i, j, k) \cap \mathfrak{m}$ for any triple $i, j, k$ with $1 \leq i<j<k \leq 4$. By (1.18), the integral in (1.23) is

$$
\leq \sum_{1 \leq i<j<k \leq 4} \int_{\mathfrak{B}(i, j, k) \cap \mathfrak{m}}\left|f\left(\gamma_{i}\right) f\left(\gamma_{j}\right) f\left(\gamma_{k}\right)\right|^{16 / 3} d \boldsymbol{\alpha} .
$$

The argument used in (1.21) gives

$$
\int_{\mathfrak{B}(i, j, k)}\left|f\left(\gamma_{i}\right) f\left(\gamma_{j}\right) f\left(\gamma_{k}\right)\right|^{5} d \boldsymbol{\alpha} \ll P^{6} .
$$

Thus, (1.23) would follow providing we are able to show

Lemma 11. For $\boldsymbol{\alpha} \in \mathfrak{B}(i, j, k) \cap \mathfrak{m}$ we have $\left|f\left(\gamma_{i}\right) f\left(\gamma_{j}\right) f\left(\gamma_{k}\right)\right| \ll$ $P^{3}(\log P)^{-\eta}$. 
Proof. This is essentially contained in Lemma 19 of Davenport and Lewis [17]. When $\boldsymbol{\alpha} \in \mathfrak{B}(i, j, k)$ we have $\gamma_{v}(\bmod 1) \in \mathfrak{E}$. Hence, by Lemma 4 , for $v=i, j, k$ there are integers $t_{v}, q_{v}$ with $\left(q_{v}, t_{v}\right)=1, q_{v} \leq P^{3 / 4}$ and $\left|q_{v} \gamma_{v}-t_{v}\right| \leq P^{-9 / 4}$. We now show that

$$
\left|f\left(\gamma_{i}\right) f\left(\gamma_{j}\right) f\left(\gamma_{k}\right)\right|>P^{3}(\log P)^{-\eta}
$$

implies $\boldsymbol{\alpha} \in \mathfrak{M}$. To see this we apply Lemma 5 and use $\kappa(q) \ll q^{\varepsilon-1 / 3}$. Then

$$
\left|f\left(\gamma_{i}\right) f\left(\gamma_{j}\right) f\left(\gamma_{k}\right)\right| \ll P^{3}\left(q_{i} q_{j} q_{k}\right)^{\varepsilon-1 / 3} \prod_{v=i, j, k}\left(1+P^{3}\left|\gamma_{v}-\frac{t_{v}}{q_{v}}\right|\right)^{-1 / 3} .
$$

Combining the last two inequalities we have

$$
q_{i} q_{j} q_{k} \ll(\log P)^{4 \eta} ; \quad\left|\gamma_{v}-\frac{t_{v}}{q_{v}}\right| \ll(\log P)^{4 \eta} P^{-3} \quad(v=i, j, k) .
$$

For $w=1,2,3$ we may write

$$
\alpha_{w}=\vartheta_{w, i} \gamma_{i}+\lambda_{w, j} \gamma_{j}+\mu_{w, k} \gamma_{k}
$$

where $\vartheta_{w, i}, \lambda_{w, j}, \mu_{w, k}$ are certain rational numbers. Thus there is an integer constant $K$, and integers $q, u_{1}, u_{2}, u_{3}$ with $q \mid K q_{i} q_{j} q_{k},\left(q, u_{1}, u_{2}, u_{3}\right)=1$ and

$$
\left|\alpha_{w}-u_{w} / q\right| \ll(\log P)^{4 \eta} P^{-3} \quad(1 \leq w \leq 3) .
$$

Since $q \ll(\log P)^{4 \eta}$ this gives $\boldsymbol{\alpha} \in \mathfrak{M}$, and establishes the lemma. Also the proof that $\mathcal{N}(\mathfrak{L})=o\left(P^{13}\right)$ is now complete.

Next we estimate the contribution from the sets $\mathfrak{G}_{i}$. We prepare for the treatment by recalling the definition of $\mathfrak{G}_{i}$, and apply Hölder's inequality. Then

$$
\mathcal{N}\left(\mathfrak{G}_{i}\right) \ll P^{9 / 4+3 \delta}\left(\int_{\mathfrak{G}_{i}}\left|f\left(\gamma_{i}\right)\right|^{3}|G(\boldsymbol{\alpha})| d \boldsymbol{\alpha}\right)^{1 / 3}\left(\int_{\mathfrak{U}}|G(\boldsymbol{\alpha})| d \boldsymbol{\alpha}\right)^{2 / 3} .
$$

The second integral here is estimated in (1.19). We shall prove that

$$
\int_{\mathfrak{G}_{i}}\left|f\left(\gamma_{i}\right)\right|^{3}|G(\boldsymbol{\alpha})| d \boldsymbol{\alpha} \ll P^{12+1 / 4+\varepsilon} ;
$$

the required bound $\mathcal{N}\left(\mathfrak{G}_{i}\right)=o\left(P^{13}\right)$ is then immediate. To attack the integral in (1.25) we decompose the indices $j, 5 \leq j \leq 22$, into two disjoint sets $\mathcal{J}_{1}, \mathcal{J}_{2}$, of nine elements each. We shall specify $\mathcal{J}_{t}$ in a moment but note already that these sets will be chosen such that $M_{\mathcal{J}_{1}}$ and $M_{\mathcal{J}_{2}}$ are still partitionable. Now define

$$
G_{t}(\boldsymbol{\alpha})=\prod_{j \in \mathcal{J}_{t}} g\left(\gamma_{j}\right)
$$


and consider the integrals

$$
I_{t}=\int_{\mathfrak{G}_{i}}\left|f\left(\gamma_{i}\right)\right|^{3}\left|G_{t}(\boldsymbol{\alpha})\right|^{2} d \boldsymbol{\alpha} \quad(t=1,2) .
$$

Before proceeding further we note that the estimation of this integral is essentially identical for all possible values of $i$, that is, $1 \leq i \leq 4$. We therefore illustrate the method in the typical case $i=4$, which is actually slightly more demanding than the cases $1 \leq i \leq 3$; and avoid unwanted complications of a notational character.

Thus concentrating on the case $i=4$, we change variables to $\left(\gamma_{1}, \gamma_{2}, \gamma_{4}\right)$ (which is a non-singular transformation), and obtain in the now familiar way

$$
\begin{aligned}
I_{t} & \ll \int_{\mathfrak{E}}\left|f\left(\gamma_{4}\right)\right|^{3} \int_{0}^{1} \int_{0}^{1}\left|G_{t}(\boldsymbol{\alpha})\right|^{2} d \gamma_{1} d \gamma_{2} d \gamma_{4} \\
& =\int_{\mathfrak{E}}\left|f\left(\gamma_{4}\right)\right|^{3} \Psi_{t}\left(\gamma_{4}\right) d \gamma_{4}, \quad \text { say. }
\end{aligned}
$$

From $\left|G_{t}(\boldsymbol{\alpha})\right|^{2} \geq 0$ we have at once $\Psi_{t}\left(\gamma_{4}\right) \geq 0$. Also, recalling (1.2) in the form

$$
\gamma_{v}=\vartheta_{v} \gamma_{1}+\lambda_{v} \gamma_{2}+\mu_{v} \gamma_{4}
$$

with integers $\vartheta_{v}, \lambda_{v}, \mu_{v}$, we also see that

$$
\Psi_{t}\left(\gamma_{4}\right)=\sum_{\substack{\boldsymbol{y}, \boldsymbol{z} \in \mathcal{A}^{9} \\ \Theta(\boldsymbol{y}, \boldsymbol{z})=\Lambda(\boldsymbol{y}, \boldsymbol{z})=0}} e\left(\gamma_{4} \mathrm{M}(\boldsymbol{y}, \boldsymbol{z})\right)=\sum_{h} \psi_{h} e\left(\gamma_{4} h\right)
$$

where $\boldsymbol{y}=\left(y_{1}, \ldots, y_{9}\right), \boldsymbol{z}=\left(z_{1}, \ldots, z_{9}\right)$,

$$
\begin{aligned}
\Theta(\boldsymbol{y}, \boldsymbol{z}) & =\Theta_{t}(\boldsymbol{y}, \boldsymbol{z})=\sum_{j \in \mathcal{J}_{t}} \vartheta_{j}\left(y_{j}^{3}-z_{j}^{3}\right), \\
\Lambda(\boldsymbol{y}, \boldsymbol{z}) & =\Lambda_{t}(\boldsymbol{y}, \boldsymbol{z})=\sum_{j \in \mathcal{J}_{t}} \lambda_{j}\left(y_{j}^{3}-z_{j}^{3}\right), \\
\mathrm{M}(\boldsymbol{y}, \boldsymbol{z})=\mathrm{M}_{t}(\boldsymbol{y}, \boldsymbol{z}) & =\sum_{j \in \mathcal{J}_{t}} \mu_{j}\left(y_{j}^{3}-z_{j}^{3}\right),
\end{aligned}
$$

and $\psi_{h}$ is the number of solutions to the simultaneous conditions

$$
\Theta_{t}(\boldsymbol{y}, \boldsymbol{z})=\Lambda_{t}(\boldsymbol{y}, \boldsymbol{z})=0, \quad \mathrm{M}_{t}(\boldsymbol{y}, \boldsymbol{z})=h, \quad \boldsymbol{x} \in \mathcal{A}^{9}, \quad \boldsymbol{y} \in \mathcal{A}^{9} .
$$

In particular, $\psi_{h} \geq 0$, and $\psi_{h}=0$ for $|h| \gg P^{3}$. We are now in a position to apply Lemma 9 to (1.26), and obtain

$$
I_{t} \ll P^{3 / 8+\varepsilon} \psi_{0}+P^{\varepsilon} \sum_{h} \psi_{h}
$$


The matrices $M_{\mathcal{J}_{t}}$ were supposed to be partitionable. Therefore the method used in (1.19) is readily adopted to show that

$$
\begin{aligned}
\psi_{0} & \ll \int_{\mathfrak{U}}\left|G_{t}(\boldsymbol{\alpha})\right|^{2} d \boldsymbol{\alpha} \\
& \ll \sum_{\substack{k, l, m \in \mathcal{J}_{t} \\
\operatorname{det} M_{k, l, m} \neq 0}} \int_{\mathfrak{U}}\left|g\left(\gamma_{k}\right) g\left(\gamma_{l}\right) g\left(\gamma_{m}\right)\right|^{6} d \gamma_{k} d \gamma_{l} d \gamma_{m} \ll P^{39 / 4+\varepsilon} .
\end{aligned}
$$

The sum over $\psi_{h}$ is more difficult to estimate, and it is here where $\mathcal{J}_{1}, \mathcal{J}_{2}$ have to be chosen carefully. Consider the matrix

$$
N=\left[\begin{array}{l}
\vartheta_{v} \\
\lambda_{v} \\
\mu_{v}
\end{array}\right]_{5 \leq v \leq 22}
$$

formed with the numbers introduced in (1.27). Since $M_{5,6, \ldots, 22}$ is partitionable, $N$ is also partionable, and we choose one partition into six disjoint submatrices $N^{(1)}, \ldots, N^{(6)}$, of rank 3 .

Let $\mathcal{V}$ be the set of all $v$ with $5 \leq v \leq 22$ and $\vartheta_{v}=\lambda_{v}=0$. Then for $v \in \mathcal{V}, \gamma_{4}$ and $\gamma_{v}$ are linearly dependent. Hence $M_{\mathcal{V} \cup\{4\}}$ has rank 1, which implies $|\mathcal{V}| \leq 4$. Also, of course, any $N^{(j)}$ may contain at most one column $\left[\begin{array}{l}\vartheta_{v} \\ \lambda_{v} \\ \mu_{v}\end{array}\right]$ with $v \in \mathcal{V}$. We may suppose that these columns are in $N^{(1)}, N^{(2)}, N^{(4)}$ and $N^{(5)}$, if any.

Next consider repetitions in the sequence $\vartheta_{v} / \lambda_{v}(5 \leq v \leq 22, v \notin \mathcal{V})$. Let $\mathcal{W}$ be a set of indices with $\vartheta_{w} / \lambda_{w}(w \in \mathcal{W})$ all equal. Then the submatrix of $N$ formed with the columns corresponding to $\mathcal{V} \cup \mathcal{W}$ has rank 2, which implies that $|\mathcal{W}| \leq 12-|\mathcal{V}|$. It is also clear that for fixed $\mathcal{W}$ any $N^{(j)}$ may contain at most two columns from $\mathcal{V} \cup \mathcal{W}$. Therefore any set of three $N^{(j)}$ contains at most six columns corresponding to a $v \in \mathcal{V} \cup \mathcal{W}$.

Now choose $\mathcal{J}_{1}$ to be the set of all $v$ corresponding to the nine columns in $N^{(1)}, N^{(2)}, N^{(3)}$, and $\mathcal{J}_{2}$ to be the set of all $v$ corresponding to the nine columns in $N^{(4)}, N^{(5)}, N^{(6)}$. The preceding arguments show that for $t=1$ and $t=2, \mathcal{J}_{t}$ contains a subset $\mathcal{K}_{t}$ with $\left|\mathcal{K}_{t}\right|=7$ such that for $v \in \mathcal{K}_{t}$ not both $\vartheta_{v}$ and $\lambda_{v}$ are zero, and such that the sequence $\vartheta_{v} / \lambda_{v}\left(v \in \mathcal{K}_{t}\right)$ contains no value more than four times. (If two $v \in \mathcal{V}$ have gone into $\mathcal{J}_{t}$ then there may be only four such equal ratios, and if not there might be five or six equal ratios but an excess can be dropped in the reduction from $\mathcal{J}_{t}$ to $\mathcal{K}_{t}$.)

We are now prepared to estimate $\sum_{h} \psi_{h}$, which we first express as an 
integral using (1.9). We have

$$
\begin{aligned}
\sum_{h} \psi_{h} & =\sum_{\substack{\boldsymbol{y}, \boldsymbol{z} \in \mathcal{A}^{9} \\
\Theta(\boldsymbol{y}, \boldsymbol{z})=\Lambda(\boldsymbol{y}, \boldsymbol{z})=0}} 1 \\
& =\int_{0}^{1} \int_{0}^{1} \prod_{j \in \mathcal{J}_{t}}\left|g\left(\vartheta_{j} \beta_{1}+\lambda_{j} \beta_{2}\right)\right|^{2} d \beta_{1} d \beta_{2} \\
& \leq P^{4} \int_{0}^{1} \int_{0}^{1} H_{t}\left(\beta_{1}, \beta_{2}\right) d \beta_{1} d \beta_{2}
\end{aligned}
$$

where

$$
H_{t}\left(\beta_{1}, \beta_{2}\right)=\prod_{j \in \mathcal{K}_{t}}\left|g\left(\vartheta_{j} \beta_{1}+\lambda_{j} \beta_{2}\right)\right|^{2} .
$$

Here we used a trivial estimate for $g(\alpha)$.

Suppose first that the sequence $\vartheta_{j} / \lambda_{j}, j \in \mathcal{K}_{t}$ contains no value more than three times. Then, by a now familiar argument, via (1.18) we infer that

$$
H_{t}\left(\beta_{1}, \beta_{2}\right) \leq \sum_{\substack{j, l \in \mathcal{K}_{t} \\ \lambda_{j} \vartheta_{l} \neq \lambda_{l} \vartheta_{j}}}\left|g\left(\vartheta_{j} \beta_{1}+\lambda_{j} \beta_{2}\right) g\left(\vartheta_{l} \beta_{1}+\lambda_{l} \beta_{2}\right)\right|^{7} .
$$

Also the change of variable $\xi_{1}=\vartheta_{j} \beta_{1}+\lambda_{j} \beta_{2}, \xi_{2}=\vartheta_{l} \beta_{1}+\lambda_{l} \beta_{2}$ is non-singular whenever $\lambda_{j} \vartheta_{l} \neq \lambda_{l} \vartheta_{j}$; and therefore, integrating the previous inequality, we deduce from Lemma 7 that

$$
\int_{0}^{1} \int_{0}^{1} H_{t}\left(\beta_{1}, \beta_{2}\right) d \beta_{1} d \beta_{2} \ll \int_{0}^{1} \int_{0}^{1}\left|g\left(\xi_{1}\right) g\left(\xi_{2}\right)\right|^{7} d \xi_{1} d \xi_{2} \ll P^{33 / 4+\varepsilon} .
$$

Now suppose that $\mathcal{K}_{t}$ contains a subset $\mathcal{L}$ with $|\mathcal{L}|=4$ such that the $\vartheta_{l} / \lambda_{l}$ with $l \in \mathcal{L}$ are all equal. Then there is a non-singular transformation $\left(\beta_{1}, \beta_{2}\right) \rightarrow\left(\xi_{1}, \xi_{2}\right)$ such that

$$
\begin{aligned}
\vartheta_{l} \beta_{1}+\lambda_{l} \beta_{2} & =\varrho_{l} \xi_{1} & & \text { if } l \in \mathcal{L}, \\
\vartheta_{k} \beta_{1}+\lambda_{k} \beta_{2} & =\varrho_{k} \xi_{1}+\sigma_{k} \xi_{2} & & \text { if } k \in \mathcal{K}_{t} \backslash \mathcal{L}
\end{aligned}
$$

where $\varrho_{j}, \sigma_{j}$ are integers with $\varrho_{l} \neq 0$ for all $l \in \mathcal{L}$ and $\sigma_{k} \neq 0$ for all $k \in \mathcal{K}_{t} \backslash \mathcal{L}$. Now

$$
\begin{aligned}
\int_{0}^{1} \int_{0}^{1} H_{t}\left(\beta_{1}, \beta_{2}\right) d \beta_{1} d \beta_{2} & \\
& \ll \int_{0}^{1} \prod_{l \in \mathcal{L}}\left|g\left(\varrho_{l} \xi_{1}\right)\right|^{2} \int_{0}^{1} \prod_{k \in \mathcal{K}_{t} \backslash \mathcal{L}}\left|g\left(\varrho_{k} \xi_{1}+\sigma_{k} \xi_{2}\right)\right|^{2} d \xi_{2} d \xi_{1} .
\end{aligned}
$$


The inner integral here equals

$$
\sum_{x_{k}, y_{k}}^{\sharp} e\left(\xi_{1} \sum_{k \in \mathcal{K}_{t} \backslash \mathcal{L}} \varrho_{k}\left(x_{k}^{3}-y_{k}^{3}\right)\right) \leq \sum_{x_{k}, y_{k}}^{\sharp} 1
$$

where $\sum_{x_{k}, y_{k}}^{\sharp}$ denotes a six-fold sum over $\left(x_{k}, y_{k}\right)_{k \in \mathcal{K}_{t} \backslash \mathcal{L}} \in \mathcal{A}^{6}$ subject to the additional constraint

$$
\sum_{k \in \mathcal{K}_{t} \backslash \mathcal{L}} \sigma_{k}\left(x_{k}^{3}-y_{k}^{3}\right)=0 .
$$

Thus, by Hölder's inequality, and Lemma 7,

$$
\begin{aligned}
\sum_{x_{k}, y_{k}}^{\sharp} 1 & =\int_{0}^{1} \prod_{k \in \mathcal{K}_{t} \backslash \mathcal{L}}\left|g\left(\sigma_{k} \alpha\right)\right|^{2} d \alpha \\
& \leq \prod_{k \in \mathcal{K}_{t} \backslash \mathcal{L}}\left(\int_{0}^{1}\left|g\left(\sigma_{k} \alpha\right)\right|^{6} d \alpha\right)^{1 / 3} \ll P^{13 / 4+\varepsilon} .
\end{aligned}
$$

This shows that the inner integral is bounded by $\ll P^{13 / 4+\varepsilon}$ uniformly in $\xi_{1}$, and now using Lemma 7 with $s=8$ together with Hölder's inequality in much the same way as before we deduce that

$$
\int_{0}^{1} \int_{0}^{1} H_{t}\left(\beta_{1}, \beta_{2}\right) d \beta_{1} d \beta_{2} \ll P^{13 / 4+\varepsilon} \int_{0}^{1} \prod_{l \in \mathcal{L}}\left|g\left(\varrho_{l} \xi_{1}\right)\right|^{2} d \xi_{1} \ll P^{13 / 4+5+\varepsilon}
$$

so that (1.33) actually holds in all cases. From (1.29), (1.30), (1.32) and (1.33) we get $I_{t} \ll P^{12+1 / 4+\varepsilon}$. Of course the left hand side of (1.25) is $\leq I_{1}+I_{2}$. This completes the proof of (1.25). A satisfactory estimate for $\mathcal{N}\left(\mathfrak{G}_{i}\right)$ is now available.

It remains to treat the sets $\mathfrak{H}_{i j}$. Again we concentrate on the case $i=2$, $j=4$ in order to avoid unnecessary notational complications, and content ourselves with the remark that all the other cases can be handled in the same way.

The method has a certain affinity to our treatment of $\mathfrak{G}_{i}$. Again we consider the matrix $N$ introduced in (1.31) together with a partition of $N$ into six disjoint submatrices $N^{(1)}, \ldots, N^{(6)}$, of rank 3 .

We need an estimate for the number of columns of $N$ with $\vartheta_{i}=0$. Let $\mathcal{J}$ be the set of all $i, 5 \leq i \leq 22$ with $\vartheta_{i}=0$. Then the matrix $M_{\{2,4\} \cup \mathcal{J}}$ has rank 2 , by a simple argument. Hence $|\mathcal{J}| \leq 10$. Also, any $N^{(j)}$ may contain at most two columns with $\vartheta_{i}=0$. Hence we may pick two $N^{(j)}$, say $N^{(1)}, N^{(2)}$, such that among the six columns in these two matrices there are at most two with $\vartheta_{i}=0$. We denote the set of all indices corresponding to the columns in $N^{(1)}, N^{(2)}$ by $\mathcal{S}$, and the set of the remaining indices from $\{5,6, \ldots, 22\}$, by $\mathcal{U}$. Then $|\mathcal{S}|=6,|\mathcal{U}|=12$, and $M_{\mathcal{S}}$ and $M_{\mathcal{U}}$ are 
partitionable matrices, by construction. Now write, for $\mathcal{T}=\mathcal{S}$ or $\mathcal{U}$,

$$
G_{\mathcal{T}}(\boldsymbol{\alpha})=\prod_{t \in \mathcal{T}} g\left(\gamma_{t}\right)
$$

so that $G(\boldsymbol{\alpha})=G_{\mathcal{S}}(\boldsymbol{\alpha}) G_{\mathcal{U}}(\boldsymbol{\alpha})$. From the definition of $\mathfrak{H}_{2,4}$ and Hölder's inequality we have

$$
\begin{aligned}
\mathcal{N}\left(\mathfrak{H}_{2,4}\right) \ll P^{3 / 2+2 \delta}\left(\int_{\mathfrak{H}_{2,4}}\left|f\left(\gamma_{2}\right) f\left(\gamma_{4}\right)\right|^{3}\left|G_{\mathcal{S}}(\boldsymbol{\alpha})\right|^{2} d \boldsymbol{\alpha}\right)^{1 / 3} & \\
& \times\left(\int_{\mathfrak{U}}\left|G_{\mathcal{S}}(\boldsymbol{\alpha})\right|^{1 / 2}\left|G_{\mathcal{U}}(\boldsymbol{\alpha})\right|^{3 / 2} d \boldsymbol{\alpha}\right)^{2 / 3} .
\end{aligned}
$$

Now

$$
\left|G_{\mathcal{S}}(\boldsymbol{\alpha})\right|^{1 / 2}\left|G_{\mathcal{U}}(\boldsymbol{\alpha})\right|^{3 / 2}=\prod_{r \in \mathcal{R}}\left|g\left(\gamma_{r}\right)\right|^{1 / 2}
$$

where $\mathcal{R}$ is the sequence formed from $\mathcal{S}$ and three copies of $\mathcal{U}$. Now, the

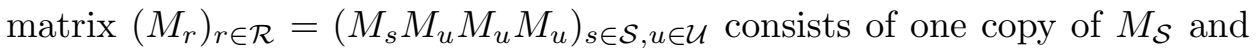
three copies of $M_{\mathcal{U}}$, and is therefore partitionable. It has 42 columns, and therefore we may use (1.18) to deduce that

$$
\left|G_{\mathcal{S}}(\boldsymbol{\alpha})\right|^{1 / 2}\left|G_{\mathcal{U}}(\boldsymbol{\alpha})\right|^{3 / 2} \leq \sum_{\substack{5 \leq k, l, m \leq 22 \\ \operatorname{det} M_{k, l, m} \neq 0}}\left|g\left(\gamma_{k}\right) g\left(\gamma_{l}\right) g\left(\gamma_{m}\right)\right|^{7}
$$

Integrating this inequality we find after a change of variable and an application of Lemma 7 that

$$
\int_{\mathfrak{U}}\left|G_{\mathcal{S}}(\boldsymbol{\alpha})\right|^{1 / 2}\left|G_{\mathcal{U}}(\boldsymbol{\alpha})\right|^{3 / 2} d \boldsymbol{\alpha} \ll P^{99 / 8+\varepsilon} .
$$

In the first integral in (1.35) we first change variables to $\gamma_{1}, \gamma_{2}, \gamma_{4}$ and recall the notation (1.27). Then, as in (1.26) we obtain

$$
\begin{aligned}
& \int_{\mathfrak{H}_{2,4}}\left|f\left(\gamma_{2}\right) f\left(\gamma_{4}\right)\right|^{3}\left|G_{\mathcal{S}}(\boldsymbol{\alpha})\right|^{2} d \boldsymbol{\alpha} \\
& \ll \int_{\mathfrak{E}} \int_{\mathfrak{E}}\left|f\left(\gamma_{2}\right) f\left(\gamma_{4}\right)\right|^{3} \int_{0}^{1}\left|G_{\mathcal{S}}(\boldsymbol{\alpha})\right|^{2} d \gamma_{1} d \gamma_{2} d \gamma_{4} \\
& \ll \int_{\mathfrak{E}} \int_{\mathfrak{E}}\left|f\left(\gamma_{2}\right) f\left(\gamma_{4}\right)\right|^{3} \Psi\left(\gamma_{2}, \gamma_{4}\right) d \gamma_{2} d \gamma_{4}, \quad \text { say. }
\end{aligned}
$$

It is clear that $\Psi\left(\gamma_{2}, \gamma_{4}\right) \geq 0$. Redefine the forms $\Theta, \Lambda, \mathrm{M}$ introduced in (1.28) by replacing the summation over $j \in \mathcal{J}_{t}$ by $j \in \mathcal{S}$ so that $\Theta, \Lambda, \mathrm{M}$ are 
forms in twelve variables now. Then

$$
\Psi\left(\gamma_{2}, \gamma_{4}\right)=\sum_{h, k} \psi_{h, k} e\left(\gamma_{2} h+\gamma_{4} k\right)
$$

where $\psi_{h, k}$ is the number of solutions to

$$
\Theta(\boldsymbol{x}, \boldsymbol{y})=0, \quad \Lambda(\boldsymbol{x}, \boldsymbol{y})=h, \quad \mathrm{M}(\boldsymbol{x}, \boldsymbol{y})=k, \quad \mathbf{x}, \boldsymbol{y} \in \mathcal{A}^{6} .
$$

We wish to use Lemma 10 now. Preparing for its application we note that

$$
\psi_{0,0}=\int_{\mathfrak{U}} \prod_{s \in \mathcal{S}}\left|g\left(\vartheta_{s} \beta_{1}+\lambda_{s} \beta_{2}+\mu_{s} \beta_{3}\right)\right|^{2} d \boldsymbol{\beta} .
$$

The matrix formed from $\left[\begin{array}{c}\vartheta_{s} \\ \lambda_{s} \\ \mu_{s}\end{array}\right]$ with $s \in \mathcal{S}$ partitions into $N^{(1)}$ and $N^{(2)}$ so that by Cauchy's inequality (or equivalently (1.18)), and two changes of variable, we find that

$$
\psi_{0,0} \ll \int_{0}^{1} \int_{0}^{1} \int_{0}^{1}\left|g\left(\xi_{1}\right) g\left(\xi_{2}\right) g\left(\xi_{3}\right)\right|^{4} d \boldsymbol{\xi} \ll P^{6+\varepsilon} .
$$

Note that $\sum_{h, k} \psi_{h, k}$ is the number of solutions to $\Theta(\boldsymbol{x}, \boldsymbol{y})=0$ with $\boldsymbol{x}, \boldsymbol{y} \in$ $\mathcal{A}^{6}$. By construction there is a set $\mathcal{V} \subset \mathcal{S}$ with $|\mathcal{V}|=4$ and $\vartheta_{v} \neq 0$ for $v \in \mathcal{V}$. Therefore, by (1.9), (1.18) and Lemma 7 ,

$$
\begin{aligned}
\sum_{h, k} \psi_{h, k} & \leq P^{4} \int_{0}^{1} \prod_{v \in \mathcal{V}}\left|g\left(\vartheta_{v} \alpha\right)\right|^{2} d \alpha \\
& \leq P^{4} \sum_{v \in \mathcal{V}} \int_{0}^{1}\left|g\left(\vartheta_{v} \alpha\right)\right|^{8} d \alpha \ll P^{9} .
\end{aligned}
$$

Next we observe that $\sum_{h} \psi_{h, 0}$ equals the number of solutions to $\Theta(\boldsymbol{x}, \boldsymbol{y})=$ $\mathrm{M}(\boldsymbol{x}, \boldsymbol{y})=0$ with $\boldsymbol{x}, \boldsymbol{y} \in \mathcal{A}^{6}$, which we may rewrite as

$$
\sum_{h} \psi_{h, 0}=\int_{0}^{1} \int_{0}^{1} \prod_{s \in \mathcal{S}}\left|g\left(\vartheta_{s} \beta_{1}+\mu_{s} \beta_{2}\right)\right|^{2} d \beta_{1} d \beta_{2} .
$$

Of course in $N^{(1)}$ there must be two columns with $\vartheta_{i} \mu_{j}-\vartheta_{j} \mu_{i} \neq 0$, otherwise we would have $\operatorname{det} N^{(1)}=0$, which is not the case. The same argument applies to $N^{(2)}$. Hence there is a subset $\mathcal{W} \subset \mathcal{S}$ with $|\mathcal{W}|=4$ such that for $w \in \mathcal{W}$ no both $\vartheta_{w}, \mu_{w}$ are zero, and such that any ratio $\vartheta_{w} / \mu_{w}$ occurs at most twice. Then, by now familiar arguments,

$$
\sum_{h} \psi_{h, 0} \leq P^{4} \int_{0}^{1} \int_{0}^{1} \prod_{w \in \mathcal{W}}\left|g\left(\vartheta_{w} \beta_{1}+\mu_{w} \beta_{2}\right)\right|^{2} d \beta_{1} \beta_{2}
$$




$$
\begin{aligned}
& \leq P^{4} \sum_{\substack{v, w \in \mathcal{W} \\
\vartheta_{w} \mu_{v} \neq \vartheta_{v} \mu_{w}}} \int_{0}^{1} \int_{0}^{1}\left|g\left(\vartheta_{w} \beta_{1}+\mu_{w} \beta_{2}\right) g\left(\vartheta_{v} \beta_{1}+\mu_{v} \beta_{2}\right)\right|^{4} d \beta_{1} d \beta_{2} \\
& \ll P^{8+\varepsilon} .
\end{aligned}
$$

By exchanging the roles of $\mu_{s}$ and $\lambda_{s}, \mathrm{M}$ and $\Lambda$, this argument also shows that

$$
\sum_{k} \psi_{0, k} \ll P^{8+\varepsilon}
$$

Collecting together we deduce from Lemma 10 that (1.37) is

$$
\ll P^{3 / 4+\varepsilon} \psi_{0,0}+P^{3 / 8+\varepsilon} \sum_{h}\left(\psi_{0, h}+\psi_{h, 0}\right)+P^{\varepsilon} \sum_{h, k} \psi_{h, k} \ll P^{9+\varepsilon} .
$$

This and (1.36) are put into (1.35), so that we can now infer

$$
\mathcal{N}\left(\mathfrak{H}_{2,4}\right) \ll P^{3 / 2+2 \delta}\left(P^{9+\varepsilon}\right)^{1 / 3}\left(P^{99 / 8+\varepsilon}\right)^{2 / 3} \ll P^{13-\delta} .
$$

The proof of (1.12) in Case I is now complete.

5. Minor arcs in case II. There are a number of new problems in the treatment of the minor arcs in Case II. Partly they arise since the matrix $M_{1,2,4}$ may well be singular, and partly because the situation is no longer "symmetric" in the three equations (0.6) in that there are many zeros in one row. However, this latter fact can also be used to good advantage, but a rather different dissection of $\mathfrak{m}$ will then be required.

We begin with the remark that the matrix $M_{5,6, \ldots, 22}$ is still partitionable. This does not follow directly from Proposition 2, but a partition can be constructed as follows. Suppose first that $R=14$. Then $c_{11}=0$. Now consider $M_{11,12, \ldots, 22}$. Here all $c_{i}$ are zero. But this matrix may not contain a submatrix of rank 1 , and consisting of six columns. Hence no value occurs more than five times in the sequence $a_{i} / b_{i}, 11 \leq i \leq 22$. Now group the indices together into six sets of two elements each such that in any of these sets one has $a_{i} b_{j} \neq a_{j} b_{i}$. Then take one of the six columns with $c_{k} \neq 0$. Thus $\operatorname{det} M_{i, j, k} \neq 0$. This partitions $M_{5,6, \ldots, 22}$.

Now suppose that $R=13$. Then $c_{11} \neq 0$. There must be two linearly independent columns $M_{i}, M_{j}$, say, with $5 \leq i, j \leq 11$ (otherwise there is a submatrix of seven columns of rank 1). If $\operatorname{det} M_{i, j, k}=0$ for all $k$ with $12 \leq k \leq 22$ then all the vectors $M_{k}=\left[\begin{array}{c}a_{k} \\ b_{k} \\ 0\end{array}\right]$ with $12 \leq k \leq 22$ would lie in the plane spanned by $M_{i}$ and $M_{j}$. However, the vectors $M_{k}$ with $12 \leq k \leq 22$ span the coordinate plane $z=0$, which gives a contradiction. Hence there is a $k$ with $12 \leq k \leq 22$ and $\operatorname{det} M_{i, j, k} \neq 0$. Now we are left with 
ten columns with $c_{i}=0$ and five columns with $c_{i} \neq 0$. Therefore further five disjoint submatrices of rank 3 can be constructed as in the case $R=14$.

We shall first consider the contribution from the sets

$$
\mathfrak{g}_{j}=\left\{\boldsymbol{\alpha} \in \mathfrak{m}:\left|f\left(\gamma_{j}\right)\right| \leq P^{3 / 4+\delta}\right\}
$$

when $j=3$ or $j=4$.

First suppose that $R=14$. Then we write

$$
G(\boldsymbol{\alpha})=G_{1}(\boldsymbol{\alpha}) G_{2}(\boldsymbol{\alpha})
$$

where

$$
G_{1}(\boldsymbol{\alpha})=\prod_{i=5}^{10} g\left(\gamma_{i}\right), \quad G_{2}(\boldsymbol{\alpha})=\prod_{i=11}^{22} g\left(\gamma_{i}\right) .
$$

Note that $G_{2}(\boldsymbol{\alpha})$ does not depend on $\alpha_{3}$. Thus we may conclude that

$$
\begin{aligned}
\mathcal{N}\left(\mathfrak{g}_{4}\right) \ll & P^{3 / 4+\delta} \int_{0}^{1} \int_{0}^{1}\left|f\left(\gamma_{1}\right) f\left(\gamma_{2}\right) G_{2}(\boldsymbol{\alpha})\right| \\
& \times \int_{0}^{1}\left|f\left(\gamma_{3}\right) G_{1}(\boldsymbol{\alpha})\right| d \alpha_{3} d \alpha_{2} d \alpha_{1} .
\end{aligned}
$$

To estimate the inner integral we observe that $\gamma_{3}=c_{3} \alpha_{3}$. Therefore, by (1.18) and Lemma 8,

$$
\begin{aligned}
\int_{0}^{1}\left|f\left(\gamma_{3}\right) G_{1}(\boldsymbol{\alpha})\right| d \alpha_{3} & \ll \sum_{i=5}^{10} \int_{0}^{c_{3}}\left|f\left(\gamma_{3}\right)\right|\left|g\left(\frac{c_{i}}{c_{3}} \gamma_{3}+a_{i} \alpha_{1}+b_{i} \alpha_{2}\right)\right|^{6} d \gamma_{3} \\
& \ll P^{4+\varepsilon}
\end{aligned}
$$

uniformly in $\alpha_{1}, \alpha_{2}$. It remains to show that

$$
I=\int_{0}^{1} \int_{0}^{1}\left|f\left(\gamma_{1}\right) f\left(\gamma_{2}\right) G_{2}(\boldsymbol{\alpha})\right| d \alpha_{2} d \alpha_{1}
$$

satisfies

$$
I \ll P^{8+3 \delta} ;
$$

then from (1.44) we deduce that

$$
\mathcal{N}\left(\mathfrak{g}_{4}\right)=o\left(P^{13}\right)
$$

as needed.

To prove (1.46) first recall that the sequence $a_{i} / b_{i}, 11 \leq i \leq 22$, contains no value more than five times. An argument similar to (1.19) and (1.20) 
now shows that

$$
\begin{aligned}
& \int_{0}^{1} \int_{0}^{1}\left|G_{2}(\boldsymbol{\alpha})\right| d \alpha_{1} d \alpha_{2} \\
& \quad \leq \sum_{\substack{11 \leq \nu, \mu \leq 22 \\
a_{\nu} b_{\mu} \neq a_{\mu} b_{\nu}}} \int_{0}^{1} \int_{0}^{1}\left|g\left(a_{\nu} \alpha_{1}+b_{\nu} \alpha_{2}\right) g\left(a_{\mu} \alpha_{1}+b_{\mu} \alpha_{2}\right)\right|^{6} d \alpha_{1} d \alpha_{2} \\
& \ll P^{13 / 2+\varepsilon}
\end{aligned}
$$

and

$$
\int_{0}^{1} \int_{0}^{1}\left|G_{2}(\boldsymbol{\alpha})\right|^{4 / 3} d \alpha_{1} d \alpha_{2} \ll P^{10} .
$$

Hence, by (1.45) and (1.48), the contribution to $I$ from the set of all $\boldsymbol{\alpha}$ where $\left|f\left(\gamma_{1}\right)\right|<P^{3 / 4+\delta}$ and $\left|f\left(\gamma_{2}\right)\right|<P^{3 / 4+\delta}$ is $\ll P^{8+2 \delta+\varepsilon}$. Now consider the contribution from the set $\mathfrak{N}$ of all $\boldsymbol{\alpha}$ where $\left|f\left(\gamma_{1}\right)\right|>P^{3 / 4+\delta}$ and $\left|f\left(\gamma_{2}\right)\right|>$ $P^{3 / 4+\delta}$. Then $\gamma_{i}(\bmod 1) \in \mathfrak{E}$ for $i=1$ and $i=2$. As in (1.21) we see via Hölder's inequality and Lemma 6 that

$$
\begin{aligned}
\iint_{\mathfrak{N}} & \left|f\left(\gamma_{1}\right) f\left(\gamma_{2}\right) G_{2}(\boldsymbol{\alpha})\right| d \alpha_{1} d \alpha_{2} \\
& \ll\left(\int_{\mathfrak{E}} \int_{\mathfrak{E}}\left|f\left(\gamma_{1}\right) f\left(\gamma_{2}\right)\right|^{4} d \gamma_{1} d \gamma_{2}\right)^{1 / 4}\left(\int_{0}^{1} \int_{0}^{1}|G(\boldsymbol{\alpha})|^{4 / 3} d \alpha_{1} d \alpha_{2}\right)^{3 / 4} \\
& \ll P^{8+\varepsilon},
\end{aligned}
$$

which is again acceptable. It remains to consider the contribution from the sets $\mathfrak{N}_{j}$ of all $\boldsymbol{\alpha}$ where $\left|f\left(\gamma_{j}\right)\right|>P^{3 / 4+\delta}$ but $\left|f\left(\gamma_{i}\right)\right| \leq P^{3 / 4+\delta}$ (where $\{i, j\}=\{1,2\})$. Pick two subscripts $k, l$ with $11 \leq k, l \leq 22$ such that $\gamma_{k}, \gamma_{l}$ are not linearly dependent on $\gamma_{1}$. For simplicity suppose that $k=11, l=12$. For all $\boldsymbol{\alpha} \in \mathfrak{N}_{1}$ we have $\gamma_{1}(\bmod 1) \in \mathfrak{E}$, and Hölder's inequality gives

$$
\iint_{\mathfrak{N}_{1}}\left|f\left(\gamma_{1}\right) f\left(\gamma_{2}\right) G_{2}(\boldsymbol{\alpha})\right| d \alpha_{1} d \alpha_{2} \ll P^{3 / 4+\delta}\left(J_{11} J_{12}\right)^{1 / 8} K^{3 / 4}
$$

where

$$
\begin{aligned}
& J_{\nu}=\int_{\mathfrak{E}}\left|f\left(\gamma_{1}\right)\right|^{4} \int_{0}^{1}\left|g\left(\gamma_{\nu}\right)\right|^{8} d \gamma_{\nu} d \gamma_{1}, \\
& K=\int_{0}^{1} \int_{0}^{1} \prod_{t=13}^{22}\left|g\left(\gamma_{t}\right)\right|^{4 / 3} d \alpha_{1} d \alpha_{2} .
\end{aligned}
$$


By Lemmata 6 and 7 we have at once $J_{\nu} \ll P^{6+\varepsilon}$. Also, any ratio $a_{l} / b_{l}$ occurs at most five times when $13 \leq l \leq 22$ so that by Lemma 7 ,

$$
K \leq \sum_{\substack{13 \leq \nu, \mu \leq 22 \\ a_{\nu} b_{\mu} \neq a_{\mu} b_{\nu}}} \int_{0}^{1} \int_{0}^{1}\left|g\left(\gamma_{\nu}\right) g\left(\gamma_{\mu}\right)\right|^{20 / 3} d \alpha_{1} d \alpha_{2} \ll P^{23 / 3+\varepsilon} .
$$

Thus the contribution from $\mathfrak{N}_{1}$ is also $\ll P^{8+2 \delta}$. The contribution from $\mathfrak{N}_{2}$ can be estimated in the same way, by interchanging the roles of $\gamma_{1}$ and $\gamma_{2}$. This proves (1.46) and (1.47).

We now estimate $\mathcal{N}\left(\mathfrak{g}_{3}\right)$. Recall the notation (1.49) and observe that $\mu_{v}=0$ if and only if $c_{v}=0$. Hence in the matrix (1.31) one has $\mu_{11}=\mu_{12}=$ $\ldots=\mu_{22}=0$. Thus, on changing variables to $\gamma_{1}, \gamma_{3}, \gamma_{4}$, the method used to estimate $\mathcal{N}\left(\mathfrak{g}_{4}\right)$ applies to $\mathcal{N}\left(\mathfrak{g}_{3}\right)$ as well, and gives of course $\mathcal{N}\left(\mathfrak{g}_{3}\right)=o\left(P^{13}\right)$.

Our next task is to estimate $\mathcal{N}\left(\mathfrak{g}_{j}\right)$ when $R=13$. Success in the above method is dependent on the large number of zeros among the $c_{j}$ 's, and we have now one more non-zero $c_{j}$. Therefore we first pick three indices, $k, l, m$, say, with $5 \leq k<l \leq 11<m \leq 22$ such that $M_{k, l, m}$ has non-zero determinant (such a matrix does in fact exist, see our proof that $M_{5, \ldots, 22}$ is partitionable when $R=13$ ). For simplicity we may suppose that $k=$ $10, l=11, m=12$. Then we decompose $G(\boldsymbol{\alpha})$ as

$$
G(\boldsymbol{\alpha})=G_{3}(\boldsymbol{\alpha}) G_{4}(\boldsymbol{\alpha}) G_{5}(\boldsymbol{\alpha})
$$

where

$$
G_{3}(\boldsymbol{\alpha})=\prod_{i=5}^{9} g\left(\gamma_{i}\right) ; \quad G_{4}(\boldsymbol{\alpha})=\prod_{i=13}^{22} g\left(\gamma_{i}\right) ; \quad G_{5}(\boldsymbol{\alpha})=g\left(\gamma_{10}\right) g\left(\gamma_{11}\right) g\left(\gamma_{12}\right) .
$$

The change of variable $\left(\alpha_{1}, \alpha_{2}, \alpha_{3}\right) \rightarrow\left(\gamma_{10}, \gamma_{11}, \gamma_{12}\right)$ is non-singular. Hence, from Lemma 7 ,

$$
\int_{\mathfrak{U}}\left|G_{5}(\boldsymbol{\alpha})\right|^{6} d \boldsymbol{\alpha} \ll P^{39 / 4+\varepsilon} .
$$

Hölder's inequality can now be used to dispose of the factor $G_{5}$ by writing

$$
\mathcal{N}\left(\mathfrak{g}_{4}\right) \ll T^{5 / 6} P^{(1 / 6)(39 / 4+\varepsilon)} P^{3 / 4+\delta}
$$

where

$$
\begin{aligned}
T & =\int_{\mathfrak{U}}\left|f\left(\gamma_{1}\right) f\left(\gamma_{2}\right) f\left(\gamma_{3}\right) G_{3}(\boldsymbol{\alpha}) G_{4}(\boldsymbol{\alpha})\right|^{6 / 5} d \boldsymbol{\alpha} \\
& =\int_{0}^{1} \int_{0}^{1}\left|f\left(\gamma_{1}\right) f\left(\gamma_{2}\right) G_{4}(\boldsymbol{\alpha})\right|^{6 / 5} \int_{0}^{1}\left|f\left(\gamma_{3}\right) G_{3}(\boldsymbol{\alpha})\right|^{6 / 5} d \alpha_{3} d \alpha_{2} d \alpha_{1}
\end{aligned}
$$


in analogy to (1.44). In the inner integral a trivial estimate for $f$ and Lemma 8 give

$$
\int_{0}^{1}\left|f\left(\gamma_{3}\right) G_{3}(\boldsymbol{\alpha})\right|^{6 / 5} d \alpha_{3} \leq P^{1 / 5} \sum_{i=5}^{9} \int_{0}^{1}\left|f\left(c_{3} \alpha_{3}\right)\right|\left|g\left(\gamma_{i}\right)\right|^{6} d \alpha_{3} \ll P^{21 / 5+\varepsilon}
$$

uniformly in $\alpha_{1}, \alpha_{2}$. This is the same method we applied to the inner integral in (1.44). Now, again by trivial estimates,

$$
\begin{aligned}
& \int_{0}^{1} \int_{0}^{1}\left|f\left(\gamma_{1}\right) f\left(\gamma_{2}\right) G_{4}(\boldsymbol{\alpha})\right|^{6 / 5} d \alpha_{1} d \alpha_{2} \\
& \quad \leq P^{2 / 5} \int_{0}^{1} \int_{0}^{1}\left|f\left(\gamma_{1}\right) f\left(\gamma_{2}\right)\right|\left|G_{4}(\boldsymbol{\alpha})\right|^{6 / 5} d \alpha_{1} d \alpha_{2}=P^{2 / 5} I^{*}, \quad \text { say. }
\end{aligned}
$$

We shall prove that

$$
I^{*} \ll P^{8+3 \delta} .
$$

Collecting together we deduce that $T \ll P^{12+3 / 5+4 \delta}$, and this gives

$$
\mathcal{N}\left(\mathfrak{g}_{4}\right) \ll P^{13-1 / 8+6 \delta},
$$

which is more than required. The bound for $I^{*}$ can be proved in much the same way as the bound for $I$ in (1.45), with $\left|G_{4}(\boldsymbol{\alpha})\right|^{6 / 5}$ taking the role of $\left|G_{2}(\boldsymbol{\alpha})\right|$. In fact, since any ratio occurs at most five times in the sequence $a_{\nu} / b_{\nu}$ when $13 \leq \nu \leq 22$, we have

$$
\left|G_{4}(\boldsymbol{\alpha})\right| \leq \sum_{\substack{13 \leq \nu, \mu \leq 22 \\ a_{\nu} b_{\mu} \neq a_{\mu} b_{\nu}}}\left|g\left(\gamma_{\nu}\right) g\left(\gamma_{\mu}\right)\right|^{5}
$$

so that we deduce from Lemma 7 that

$$
\begin{aligned}
& \int_{0}^{1} \int_{0}^{1}\left|G_{4}(\boldsymbol{\alpha})\right|^{6 / 5} d \boldsymbol{\alpha} \ll P^{13 / 2+\varepsilon}, \\
& \int_{0}^{1} \int_{0}^{1}\left|G_{4}(\boldsymbol{\alpha})\right|^{(4 / 3)(6 / 5)} d \boldsymbol{\alpha} \ll P^{10},
\end{aligned}
$$

which serve as surrogates for (1.48) and (1.49). Then, following our treatment of $I$, it only remains to estimate the contribution from $\mathfrak{N}_{1}$ and $\mathfrak{N}_{2}$. Here a little more care is required in picking the numbers $k, l$. It is clear that among the numbers $a_{13} / b_{13}, \ldots, a_{22} / b_{22}$ a ratio with $b_{j}=0$ can occur at most four times (otherwise, since $b_{1}=0$, there would be a $6 \times 3$ submatrix of $M$ of rank 1 , which is not the case). All the other ratios $a_{v} / b_{v}$ may occur five times, but for these we find automatically that $\gamma_{v}$ is linearly independent of $\gamma_{1}$. Hence, by picking $\gamma_{l}, \gamma_{k}$ from the largest blocks of equal 
ratios with $b_{v} \neq 0$, we can arrange that in the sequence $a_{v} / b_{v}, 13 \leq v \leq 22$, $v \neq k, l$, any ratio occurs at most four times. For simplicity we may suppose that $k=13, l=14$. Then we have

$$
\int_{\mathfrak{N}_{1}}\left|f\left(\gamma_{1}\right) f\left(\gamma_{2}\right)\right|\left|G_{4}(\boldsymbol{\alpha})\right|^{6 / 5} d \boldsymbol{\alpha} \ll\left(J_{13} J_{14}\right)^{1 / 8}\left(K^{*}\right)^{3 / 4} .
$$

Here $J_{k}$ is given by (1.50), and

$$
K^{*}=\int_{0}^{1} \int_{0}^{1}\left(\prod_{v=15}^{22}\left|g\left(\gamma_{v}\right)\right|^{(4 / 3)(6 / 5)}\right)\left(\left|g\left(\gamma_{13}\right) g\left(\gamma_{14}\right)\right|^{4 / 15}\right) d \boldsymbol{\alpha} .
$$

By Hölder's inequality we find, as in (1.51), that $K^{*} \ll P^{23 / 3+\varepsilon}$. This completes the proof of (1.52). It must now suffice to remark that the treatment carries over to the set $\mathfrak{g}_{3}$ just as in the case $R=14$.

We now distinguish two cases, whether $M_{1,3,4}$ is singular or not (that is, whether $b_{4}=0$ or not).

First suppose that $\operatorname{det} M_{1,3,4} \neq 0$. Then $M_{i, j, k}$ is non-singular for all $1 \leq i<j<k \leq 4$, just as in Case I. In particular, the change of variable $\boldsymbol{\alpha} \rightarrow\left(\gamma_{1}, \gamma_{3}, \gamma_{4}\right)$ is non-singular. Slightly digressing from earlier usage we now write

$$
\gamma_{v}=\vartheta_{v} \gamma_{1}+\lambda_{v} \gamma_{3}+\mu_{v} \gamma_{4}
$$

and consider the associated matrix $N$ defined in (1.31). This matrix is partitionable since $M_{5,6, \ldots, 22}$ is partitionable. By Proposition 2, the largest $n \times 3$ submatrix of rank 2 consists of $n \leq 12$ columns. Let $\mathcal{V}$ be the set of all $v$ with $5 \leq v \leq 22$ and $\vartheta_{v} \neq 0$. By the condition on rank 2 submatrices we have at once $|\mathcal{V}| \geq 6$.

If $|\mathcal{V}|=6$ or $|\mathcal{V}|=7$ then we are exactly in the same situation we were faced with in estimating $\mathcal{N}\left(\mathfrak{g}_{3}\right)$ and $\mathcal{N}\left(\mathfrak{g}_{4}\right)$. Hence the arguments given above apply here as well, and we immediately have the bound

$$
\mathcal{N}\left(\mathfrak{g}_{1}\right)=o\left(P^{13}\right) .
$$

From (1.41) and the definitions in $\S 4$ we easily deduce that

$$
\mathfrak{m}=\mathfrak{g}_{3} \cup \mathfrak{g}_{4} \cup \mathfrak{g}_{1} \cup \mathfrak{K}_{2} \cup \mathfrak{L} .
$$

Now recall the treatment of $\mathfrak{K}_{2}$ in $\S 4$. The only ingredients are (1.20) and (1.21), and these estimates hold providing that $\operatorname{det} M_{1,3,4} \neq 0$ and $M_{5,6, \ldots, 22}$ is partitionable. Thus (1.22) holds here as well. The treatment of the set $\mathfrak{L}$ required also (1.20), which is available here, and Lemma 11 and (1.24). Here we needed that all $3 \times 3$ submatrices of $M_{1,2,3,4}$ have rank 3 , which is our initial hypothesis currently. Hence, as in $\S 4$ we have $\mathcal{N}(\mathfrak{L})=o\left(P^{13}\right)$, and this finally gives $\mathcal{N}(\mathfrak{m})=o\left(P^{13}\right)$.

Now suppose that $|\mathcal{V}| \geq 8$. Recall that the treatment of $\mathfrak{H}_{3,4}$ in $\S 4$ depended on the facts that $|\mathcal{V}| \geq 8$, that $M_{5,6, \ldots, 22}$ is partitionable, and that 
$\operatorname{det} M_{1,3,4} \neq 0$. All these conditions being fulfilled here, we may refer to $\S 4$ for the estimate

$$
\mathcal{N}\left(\mathfrak{H}_{3,4}\right)=o\left(P^{13}\right)
$$

Now note that

$$
\mathfrak{m}=\mathfrak{g}_{3} \cup \mathfrak{g}_{4} \cup \mathfrak{H}_{3,4} \cup \mathfrak{K}_{1} \cup \mathfrak{K}_{2} \cup \mathfrak{L} .
$$

We saw already that the sets $\mathfrak{K}_{i}$ and $\mathfrak{L}$ can be treated as in $\S 4$, so that again we arrive at $\mathcal{N}(\mathfrak{m})=o\left(P^{13}\right)$. This establishes (1.12) in Case II when $M_{1,3,4}$ is non-singular.

It remains to complete the estimation of $\mathcal{N}(\mathfrak{m})$ when $M_{1,3,4}$ is singular. In this case we wish to change variables to $\gamma_{2}, \gamma_{3}, \gamma_{4}$, which is possible since $M_{2,3,4}$ has non-vanishing determinant. Thus, for $v \geq 5$ we write

$$
\gamma_{v}=\vartheta_{v} \gamma_{2}+\lambda_{v} \gamma_{3}+\mu_{v} \gamma_{4}
$$

Also we may write

$$
\gamma_{1}=d_{3} \gamma_{3}+d_{4} \gamma_{4}
$$

with $d_{k}$ non-zero rationals. Thus, the matrix of coefficients of the linear forms $\gamma_{v}$ in $\gamma_{2}, \gamma_{3}, \gamma_{4}$ is of the shape

$$
\left[\begin{array}{ccccccc}
0 & 1 & 0 & 0 & \vartheta_{5} & \ldots & \vartheta_{22} \\
d_{3} & 0 & 1 & 0 & \lambda_{5} & \ldots & \lambda_{22} \\
d_{4} & 0 & 0 & 1 & \mu_{5} & \ldots & \mu_{22}
\end{array}\right] .
$$

As before let $\mathcal{V}$ be the set of all $v$ with $5 \leq v \leq 22$ and $\vartheta_{v} \neq 0$. Any row in (1.53) may contain at most 14 zeros (since $R \leq 14$ ). Hence $|\mathcal{V}| \geq 7$.

We wish to estimate the contribution from the set

$$
\mathfrak{h}_{2}=\left\{\boldsymbol{\alpha} \in \mathfrak{m}:\left|f\left(\gamma_{2}\right)\right| \leq P^{3 / 4+\delta},\left|f\left(\gamma_{l}\right)\right| \geq P^{3 / 4+\delta}(l=3,4)\right\}
$$

first. Here the argument again bifurcates according to $|\mathcal{V}|=7$ or $|\mathcal{V}| \geq 8$.

First suppose that $|\mathcal{V}|=7$. For notational simplicity we shall also suppose that $\mathcal{V}=\{5,6, \ldots, 11\}$. Then we decompose $G$ into

$$
G(\boldsymbol{\alpha})=G_{6}(\boldsymbol{\alpha}) G_{7}(\boldsymbol{\alpha})
$$

where

$$
G_{6}(\boldsymbol{\alpha})=\prod_{i=5}^{11} g\left(\gamma_{i}\right), \quad G_{7}(\boldsymbol{\alpha})=\prod_{i=12}^{22} g\left(\gamma_{i}\right),
$$

and observe that the definition of $\mathfrak{h}_{2}$ leads to the estimate

$$
\begin{aligned}
\mathcal{N}\left(\mathfrak{h}_{2}\right) \ll & P^{3 / 4+\delta} \int_{\mathfrak{E}} \int_{\mathfrak{E}}\left|f\left(\gamma_{1}\right) f\left(\gamma_{3}\right) f\left(\gamma_{4}\right) G_{7}(\boldsymbol{\alpha})\right| \\
& \times \int_{0}^{1}\left|G_{6}(\boldsymbol{\alpha})\right| d \gamma_{2} d \gamma_{3} d \gamma_{4} .
\end{aligned}
$$


Now consider the inner integral here. We have, by (1.18) and Schwarz's inequality,

$$
\begin{aligned}
\int_{0}^{1}\left|G_{6}(\boldsymbol{\alpha})\right| d \gamma_{2} & \leq \sum_{i=5}^{11} \int_{0}^{1}\left|g\left(\gamma_{i}\right)\right|^{7} d \gamma_{2} \\
& \leq \sum_{i=5}^{11}\left(\int_{0}^{1}\left|g\left(\gamma_{i}\right)\right|^{6} d \gamma_{2}\right)^{1 / 2}\left(\int_{0}^{1}\left|g\left(\gamma_{i}\right)\right|^{8} d \gamma_{2}\right)^{1 / 2} .
\end{aligned}
$$

But, for $s=3$ or $s=4$,

$$
\begin{aligned}
\int_{0}^{1}\left|g\left(\gamma_{i}\right)\right|^{2 s} d \gamma_{2} & =\sum_{\substack{x_{1}^{3}+\ldots+x_{s}^{3}=y_{1}^{3}+\ldots+y_{s}^{3} \\
x_{i}, y_{i} \in \mathcal{A}}} e\left(\sum_{i=1}^{s}\left(\lambda_{i} \gamma_{3}+\mu_{i} \gamma_{4}\right)\left(x_{i}^{3}-y_{i}^{3}\right)\right) \\
& \leq \sum_{\substack{x_{1}^{3}+\ldots+x_{s}^{3}=y_{1}^{3}+\ldots+y_{s}^{3} \\
x_{i}, y_{i} \in \mathcal{A}}} 1=\int_{0}^{1}|g(\alpha)|^{2 s} d \alpha .
\end{aligned}
$$

Hence, by Lemma 7, (1.54) and Hölder's inequality,

$$
\begin{aligned}
\mathcal{N}\left(\mathfrak{h}_{2}\right) \ll & P^{3 / 4+33 / 8+2 \delta} \int_{\mathfrak{E}} \int_{\mathfrak{E}}\left|f\left(\gamma_{3}\right) f\left(\gamma_{4}\right) f\left(\gamma_{1}\right) G_{7}(\boldsymbol{\alpha})\right| d \gamma_{3} d \gamma_{4} \\
\ll & P^{39 / 8+2 \delta}\left(\int_{\mathfrak{E}} \int_{\mathfrak{E}}\left|f\left(\gamma_{3}\right) f\left(\gamma_{4}\right)\right|^{4} d \gamma_{3} d \gamma_{4}\right)^{1 / 4} \\
& \times\left(\int_{0}^{1} \int_{0}^{1}\left|f\left(\gamma_{1}\right) G_{7}(\boldsymbol{\alpha})\right|^{4 / 3} d \gamma_{3} d \gamma_{4}\right)^{3 / 4} .
\end{aligned}
$$

By a now familiar argument the sequence $d_{3} / d_{4}, \lambda_{12} / \mu_{12}, \ldots, \lambda_{22} / \mu_{22}$ does not contain a value occurring more than five times. Hence

$$
\left|f\left(\gamma_{1}\right) G_{7}(\boldsymbol{\alpha})\right|^{4 / 3} \leq\left|f\left(\gamma_{1}\right) g\left(\gamma_{t}\right)\right|^{8}+\sum_{\substack{12 \leq i<j \leq 22 \\ \lambda_{i} \mu_{j} \neq \lambda_{j} \mu_{i}}}\left|g\left(\gamma_{i}\right) g\left(\gamma_{j}\right)\right|^{8}
$$

for some $t$ such that $\gamma_{t}$ and $\gamma_{1}$ are linearly independent. Then as usual we deduce from Lemma 7 and the bound (Vaughan [24], Theorem 2)

$$
\int_{0}^{1}|f(\alpha)|^{8} d \alpha \ll P^{5}
$$

that

$$
\int_{0}^{1} \int_{0}^{1}\left|f\left(\gamma_{1}\right) G_{7}(\boldsymbol{\alpha})\right|^{4 / 3} d \gamma_{3} d \gamma_{4} \ll P^{10}
$$


The first integral in (1.55) is estimated by Lemma 6 , and we arrive at

$$
\mathcal{N}\left(\mathfrak{h}_{2}\right) \ll P^{39 / 8+2 \delta}\left(P^{2+\varepsilon}\right)^{1 / 4}\left(P^{10}\right)^{3 / 4} \ll P^{13-\delta},
$$

which is acceptable.

Now suppose that $|\mathcal{V}| \geq 8$, choose a partition of the submatrix $N$ (as defined in (1.31)) of the matrix (1.53), and write this partition as $N^{(1)}, \ldots, N^{(6)}$, where as on earlier occasions the $N^{(j)}$ are disjoint $3 \times 3$ submatrices of $N$, of rank 3. From $|\mathcal{V}| \geq 8$ we deduce that there must be two $N^{(j)}$ which have at least two columns with $\vartheta_{k} \neq 0$, say $N^{(1)}, N^{(2)}$. Denote the set of all indices corresponding to the columns in $N^{(1)}$ and $N^{(2)}$ by $\mathcal{D}$, and the other indices in $\{5,6, \ldots, 22\}$ by $\mathcal{E}$. Then, using the notation introduced in (1.34), we find from Schwarz's inequality that

$$
\begin{aligned}
& \mathcal{N}\left(\mathfrak{h}_{2}\right) \ll P^{3 / 4+\delta}\left(\int_{\mathfrak{E}} \int_{\mathfrak{E}}\left|f\left(\gamma_{3}\right) f\left(\gamma_{4}\right) f\left(\gamma_{1}\right)\right|^{2}\right. \\
&\left.\int_{0}^{1}\left|G_{\mathcal{D}}(\boldsymbol{\alpha})\right|^{2} d \gamma_{2} d \gamma_{3} d \gamma_{4}\right)^{1 / 2} \\
& \times\left(\int_{\mathfrak{U}}\left|G_{\mathcal{E}}(\boldsymbol{\alpha})\right|^{2} d \boldsymbol{\alpha}\right)^{1 / 2} .
\end{aligned}
$$

By construction the submatrix of $N$ formed with the columns from $\mathcal{E}$ is partitionable. Hence the second integral is readily seen to be $\ll P^{15}$. Thus

$$
\mathcal{N}\left(\mathfrak{h}_{2}\right) \ll P^{15 / 2+3 / 4+\delta} J^{1 / 2}
$$

where $J$ denotes the first triple integral in (1.57).

Let $\mathfrak{C}$ be the set of all $\left(\gamma_{3}, \gamma_{4}\right) \in \mathfrak{E} \times \mathfrak{E}$ where $\left|f\left(\gamma_{1}\right)\right| \geq P^{3 / 4+\delta}$, and let $\mathfrak{D}$ be the set of all such $\left(\gamma_{3}, \gamma_{4}\right)$ where the opposite inequality $\left|f\left(\gamma_{1}\right)\right|<P^{3 / 4+\delta}$ holds. Then $J=J_{\mathfrak{C}}+J_{\mathfrak{D}}$ where $J_{\mathfrak{B}}$ denotes the contribution to $J$ arising from a subset $\mathfrak{B} \subset \mathfrak{E} \times \mathfrak{E}$. Note that the two-dimensional changes of variable $\left(\gamma_{3}, \gamma_{4}\right) \rightarrow\left(\gamma_{1}, \gamma_{3}\right)$ and $\left(\gamma_{3}, \gamma_{4}\right) \rightarrow\left(\gamma_{1}, \gamma_{4}\right)$ are both non-singular, and that $\left(\gamma_{3}, \gamma_{4}\right) \in \mathfrak{C}$ implies that $\gamma_{1}(\bmod 1) \in \mathfrak{E}$. The variable $\gamma_{2}$ being unaffected by these transformations we deduce via (1.18) that

$$
J_{\mathfrak{C}} \ll J_{13}+J_{14}+J_{34}
$$

where

$$
J_{k l}=\int_{\mathfrak{E}} \int_{\mathfrak{E}}\left|f\left(\gamma_{k}\right) f\left(\gamma_{l}\right)\right|^{3} \Psi_{k, l}\left(\gamma_{k}, \gamma_{l}\right) d \gamma_{k} d \gamma_{l}
$$

with

$$
\Psi_{k, l}\left(\gamma_{k}, \gamma_{l}\right)=\int_{0}^{1}\left|G_{\mathcal{D}}(\boldsymbol{\alpha})\right|^{2} d \gamma_{2} .
$$

For $d \in \mathcal{D}$ we write

$$
\gamma_{d}=\vartheta_{d} \gamma_{2}+\lambda_{d}^{(k l)} \gamma_{k}+\mu_{d}^{(k l)} \gamma_{l}
$$


and then see that

$$
\Psi_{k, l}\left(\gamma_{k}, \gamma_{l}\right)=\sum_{h_{1}, h_{2}} \psi_{k, l}\left(h_{1}, h_{2}\right) e\left(h_{1} \gamma_{k}+h_{2} \gamma_{l}\right)
$$

where $\psi_{k, l}\left(h_{1}, h_{2}\right)$ is the number of solutions to

$$
\begin{gathered}
\sum_{d \in \mathcal{D}} \vartheta_{d}\left(x_{d}^{3}-y_{d}^{3}\right)=0, \\
\sum_{d \in \mathcal{D}} \lambda_{d}^{(k l)}\left(x_{d}^{3}-y_{d}^{3}\right)=h_{1}, \\
\sum_{d \in \mathcal{D}} \mu_{d}^{(k l)}\left(x_{d}^{3}-y_{d}^{3}\right)=h_{2} .
\end{gathered}
$$

Recall that at least four numbers $\vartheta_{d}$ are non-zero. Hence the integrals $J_{k l}$ are the same as the integrals occurring on the right hand side of (1.37). Thus, as in (1.40) we see that $J_{k l} \ll P^{9+\varepsilon}$, and hence $J_{\mathfrak{C}} \ll P^{9+\varepsilon}$.

For the treatment of $\mathfrak{D}$ we imitate the proof of Lemma 10. In the notation introduced there, and bringing in the upper bound for $f\left(\gamma_{1}\right)$ we obtain the initial estimate

$J_{\mathfrak{D}} \ll P^{3 / 2+2 \delta-2} \sum_{q_{3} \leq P^{3 / 4}} \sum_{q_{4} \leq P^{3 / 4}} \kappa\left(q_{3}\right)^{2} \kappa\left(q_{4}\right)^{2} \sum_{h_{3}, h_{4}}\left|c_{q_{3}}\left(h_{3}\right) c_{q_{4}}\left(h_{4}\right)\right| \psi\left(h_{3}, h_{4}\right)$.

Here we have dropped an unimportant double index from $\psi$. The contribution from terms with $h_{3}=h_{4}=0$ is

$$
\ll P^{3 / 2+2 \delta-2} \sum_{q_{3} \leq P^{3 / 4}} \sum_{q_{4} \leq P^{3 / 4}} q_{3} q_{4} \kappa\left(q_{3}\right)^{2} \kappa\left(q_{4}\right)^{2} \psi(0,0) \ll P^{7+3 \delta}
$$

by (1.38) and the easy bound

$$
\sum_{q \leq X} \kappa(q)^{2} \ll X^{\varepsilon}
$$

For the contribution from terms with $h_{3}=0$ and $h_{4} \neq 0$ we use $\kappa\left(q_{4}\right)^{2} \ll$ $q_{4}^{\varepsilon-2 / 3} \ll P^{1 / 4+\varepsilon} q_{4}^{-1}$ for $q_{4} \leq P^{3 / 4}$, and may now proceed as in the proof of Lemma 10 . We find that these terms contribute

$$
\begin{aligned}
& \ll P^{3 / 2+3 \delta-2+1 / 4} \sum_{q_{3} \leq P^{3 / 4}} q_{3} \kappa\left(q_{3}\right)^{2} \sum_{q_{4} \leq P^{3 / 4}} q_{4}^{-1} \sum_{h_{4} \neq 0}\left|c_{q_{4}}\left(h_{4}\right)\right| \psi\left(0, h_{4}\right) \\
& \ll P^{1 / 2+4 \delta} \sum_{h_{4} \neq 0} \psi\left(0, h_{4}\right) \ll P^{8+1 / 2+5 \delta} \ll P^{9} .
\end{aligned}
$$

In the final estimate we used (1.40). Of course terms with $h_{4}=0, h_{3} \neq 0$ can be treated by the same method. In much the same way we also see that 
the contribution from terms with $h_{3} h_{4} \neq 0$ is

$$
\begin{aligned}
& \ll P^{3 / 2-2+3 \delta+1 / 2} \sum_{q_{3}, q_{4} \leq P^{3 / 4}}\left(q_{3} q_{4}\right)^{-1} \sum_{h_{3} h_{4} \neq 0}\left|c_{q_{3}}\left(h_{3}\right) c_{q_{4}}\left(h_{4}\right)\right| \psi\left(h_{3}, h_{4}\right) \\
& \ll P^{4 \delta} \sum_{h_{3}, h_{4}} \psi\left(h_{3}, h_{4}\right) \ll P^{9+5 \delta} .
\end{aligned}
$$

This follows via (1.38). Hence we now have $J_{\mathfrak{D}} \ll P^{9+5 \delta}$, and the same bound therefore holds for $J$. This is put into (1.58) to infer that

$$
\mathcal{N}\left(\mathfrak{h}_{2}\right) \ll P^{13-1 / 4+6 \delta} .
$$

Hence (1.56) is now verified in all cases.

The remaining part of $\mathfrak{m}$ is easier to deal with. We observe that

$$
\mathfrak{m}=\mathfrak{g}_{3} \cup \mathfrak{g}_{4} \cup \mathfrak{h}_{2} \cup \mathfrak{K}_{1} \cup \mathfrak{L} ;
$$

and since $M_{2,3,4}$ has non-vanishing determinant and $M_{5,6, \ldots, 22}$ is partitionable, the treatment of $\mathfrak{K}_{1}$ in $\S 4$ applies here as well. Thus it remains to estimate the contribution from $\mathfrak{L}$, which is a bit harder than in Case I. The method from $\S 4$ is readily adopted to show that

$$
\int_{\mathfrak{L}}\left|f\left(\gamma_{i}\right) f\left(\gamma_{j}\right) f\left(\gamma_{k}\right)\right|^{24 / 5} d \boldsymbol{\alpha}=o\left(P^{72 / 5-9}\right)=o\left(P^{17 / 5}\right)
$$

providing $1 \leq i<j<k \leq 4$ and $\operatorname{det} M_{i, j, k} \neq 0$.

When $R=14$ we choose $i=1, j=2, k=4$, and when $R=13$ we choose $i=2, j=3, k=4$. Then, by (1.59) and Hölder's inequality,

$$
\mathcal{N}(\mathfrak{L}) \ll\left(o\left(P^{17 / 5}\right)\right)^{5 / 24}\left(\int_{\mathfrak{U}}\left|f\left(\gamma_{l}\right) G(\boldsymbol{\alpha})\right|^{24 / 19} d \boldsymbol{\alpha}\right)^{19 / 24}
$$

where $l=4$ when $R=14$ and $l=1$ when $R=13$. Now, by (1.18),

$$
\left|f\left(\gamma_{l}\right) G(\boldsymbol{\alpha})\right|^{24 / 19} \leq|G(\boldsymbol{\alpha})|^{4 / 3}+\sum_{j=5}^{22}\left|f\left(\gamma_{l}\right) \prod_{\substack{i \neq j \\ i=5}}^{22} g\left(\gamma_{i}\right)\right|^{4 / 3} .
$$

Thus, providing that any $18 \times 3$ submatrix of $M_{l, 5,6, \ldots, 22}$ is partitionable, we may integrate the previous inequality and find in much the same way as in (1.20) that

$$
\int_{\mathfrak{U}}\left|f\left(\gamma_{l}\right) G(\boldsymbol{\alpha})\right|^{24 / 19} d \boldsymbol{\alpha} \ll P^{15} .
$$

This implies that $\mathcal{N}(\mathfrak{L})=o\left(P^{13}\right)$, and it remains to show that any $18 \times 3$ submatrix of $M_{l, 5,6, \ldots, 22}$ is partitionable. Write, for brevity, $\mathcal{U}=\{l, 5,6, \ldots, 22\}$, and define $M_{j}^{\prime}=M_{\mathcal{U} \backslash\{j\}}$ to have a shorthand for the submatrix of $M_{\mathcal{U}}$ with the $j$ th column taken out. 
First consider the case $R=14, l=4$. For $M_{l}^{\prime}=M_{4}^{\prime}$ there is nothing to prove. If $5 \leq j \leq 10$ then $M_{j}^{\prime}$ contains exactly six columns with $c_{j} \neq 0$ and twelve columns with $c_{j}=0$. Now the method used at the beginning of this section is readily adopted to construct a partition of $M_{j}^{\prime}$. If $11 \leq j \leq 22$ then $M_{j}^{\prime}$ contains exactly seven columns with $c_{j} \neq 0$ and eleven columns with $c_{j}=0$. Again the method from the beginning of this section (this time from the consideration of the case $R=13$ there) can be used to find a partition.

Now let $R=13, l=1$. Again for $M_{1}^{\prime}$ there is nothing to show. But, when $5 \leq j \leq 11$, then $M_{j}^{\prime}$ contains exactly six columns with $c_{j} \neq 0$, and when $12 \leq j \leq 22$, then $M_{j}^{\prime}$ contains exactly seven columns with $c_{j} \neq 0$. Thus we may again refer to the beginning of this section for a method to find a partition.

This establishes (1.12) in case II.

6. Asymptotics on the major arcs. The treatment of the major arcs is by standard endgame technique in the Hardy-Littlewood method. We can closely follow Davenport and Lewis [17], and the argument is only given in outline.

Let $\alpha_{j}=t_{j} / q+\beta_{j}$. Then, by (1.2),

$$
\begin{aligned}
\gamma_{v} & =\frac{a_{v} t_{1}+b_{v} t_{2}+c_{v} t_{3}}{q}+a_{v} \beta_{1}+b_{v} \beta_{2}+c_{v} \beta_{3} \\
& =\frac{d_{v}}{q_{v}}+\eta_{v}, \quad \text { say, }
\end{aligned}
$$

with integers $d_{v}, q_{v}$ satisfying $\left(d_{v}, q_{v}\right)=1$ and $q_{v} \mid q$. Then, from Lemma 5 we have at once

$$
f\left(\gamma_{v}\right)=q_{v}^{-1} S\left(q_{v}, d_{v}\right) w\left(\eta_{v}\right)+O\left(P^{\varepsilon}\right)
$$

for $1 \leq v \leq 4$ and $\boldsymbol{\alpha} \in \mathfrak{M}(q, \boldsymbol{t})$. Also, for these $\boldsymbol{\alpha}$ we have

$$
g\left(\gamma_{v}\right)=q_{v}^{-1} S\left(q_{v}, d_{v}\right) w^{*}\left(\eta_{v}\right)+O\left(P(\log P)^{-10 \delta}\right)
$$

where

$$
w^{*}(\beta)=\frac{1}{3} \int_{P^{3}}^{(C P)^{3}} \alpha^{-2 / 3} \varrho\left(\frac{\log \alpha}{\eta \log P}\right) e(\alpha \beta) d \alpha
$$

and $\varrho(\alpha)$ is Dickman's function (for a precise definition see [8]) of which we only need to know that it is continuous, positive and bounded for $\alpha \in[1, C]$. A proof of (1.61) is given in $\S 13$ of Brüdern [6]. The measure of $\mathfrak{M}$ is $\ll(\log P)^{9 \delta} P^{-9}$, and with the trivial bounds $w(\beta) \ll P, w^{*}(\beta) \ll P$ and $q^{-1} S(q, d) \ll 1$ we see that

$$
\mathcal{N}(\mathfrak{M})=\mathfrak{S}_{1} J+o\left(P^{13}\right)
$$


where

$$
\begin{aligned}
J & =\int_{\mathfrak{M}(1,0,0,0)} w\left(\eta_{1}\right) w\left(\eta_{2}\right) w\left(\eta_{3}\right) w\left(\eta_{4}\right) \prod_{j=5}^{22} w^{*}\left(\eta_{j}\right) d \beta_{1} d \beta_{2} d \beta_{3}, \\
\mathfrak{S}_{1} & =\sum_{q \leq(\log P)^{\delta}} \sum_{t}^{*}\left(q_{1} \ldots q_{22}\right)^{-2} S\left(q_{1}, d_{1}\right) \ldots S\left(q_{22}, d_{22}\right),
\end{aligned}
$$

and where $\sum_{t}^{*}$ denotes a sum over $1 \leq t_{1}, t_{2}, t_{3} \leq q$ with $\left(t_{1}, t_{2}, t_{3}, q\right)=1$. The method of proof of Lemma 30 of Davenport and Lewis [17] is readily adopted to show that

$$
J=\Gamma P^{13}+o\left(P^{13}\right)
$$

for some constant $\Gamma>0$.

Now we complete the partial singular series (1.62). Here we require the bound

$$
\sum_{t}^{*}\left(q_{1} \ldots q_{22}\right)^{-1 / 3} \ll q^{\varepsilon-4 / 3},
$$

the numbers $q_{j}$ being given by (1.60). To see this note that $M_{2,3, \ldots, 22}$ is partitionable. Hence, by (1.18),

$$
\sum_{t}^{*}\left(q_{1} \ldots q_{22}\right)^{-1 / 3} \leq \sum_{\substack{2 \leq i<j<k \leq 22 \\ \operatorname{det} M_{i, j, k} \neq 0}} \sum_{t}^{*}\left(q_{i} q_{j} q_{k}\right)^{-7 / 3} .
$$

Now the method of proof of Lemma 23 in Davenport and Lewis [17] is readily adopted (there are only obvious modifications in the exponent to be made) to bound the inner sum by $q^{\varepsilon-4 / 3}$. This gives (1.65).

The bound $q_{v}^{-1} S\left(q_{v}, d_{v}\right) \ll q_{v}^{\varepsilon-1 / 3}$ is implicit in (1.17). We also have

$$
q_{v}^{-1} S\left(q_{v}, d_{v}\right)=q^{-1} S\left(q, a_{v} t_{1}+b_{v} t_{2}+c_{v} t_{3}\right)
$$

as is readily checked from (1.15). Hence, from (1.65) and (1.63) we deduce that

$$
\mathfrak{S}_{1}=\mathfrak{S}+o(1)
$$

where

$$
\mathfrak{S}=\sum_{q=1}^{\infty} q^{-22} \sum_{t}^{*} \prod_{i=1}^{22} S\left(q, a_{i} t_{1}+b_{i} t_{2}+c_{i} t_{3}\right) .
$$

Combining (1.62), (1.64) and (1.66) we arrive at (1.13).

By (1.65), the series (1.67) is absolutely convergent. The proof of Lemma 31 in Davenport and Lewis [17] applies here as well, with their reference to their Lemma 23 replaced by our (1.65), which suffices. We restate this lemma here for convenience. 
LEMMA 12. Suppose the equations (0.6) have a non-singular p-adic solution for all primes $p$. Then $\mathfrak{S}>0$.

Under the hypothesis of Lemma 12 we have $\mathcal{N} \gg P^{13}$ from (1.12) and (1.13). In particular, this proves Theorem 1A.

CHAPTER 2

\section{p-ADIC ARGUMENTS}

1. Introduction to $p$-adic solutions. We label the three additive cubic equations as

$$
\begin{aligned}
& F(\boldsymbol{x})=a_{1} x_{1}^{3}+\ldots+a_{N} x_{N}^{3}=0, \\
& G(\boldsymbol{x})=b_{1} x_{1}^{3}+\ldots+b_{N} x_{N}^{3}=0, \\
& H(\boldsymbol{x})=c_{1} x_{1}^{3}+\ldots+c_{N} x_{N}^{3}=0
\end{aligned}
$$

with integer coefficients, where $N \geq 22$. The purpose of this chapter is to prove Theorem 2 which we restate here for convenience.

Theorem 2. Suppose that $N \geq 22$. Then the equations (2.1) have a nontrivial p-adic solutions except (possibly) for $p=3,7,13,19,31,37$ and 43.

Recall that Davenport and Lewis [15] showed that the congruences

$$
\begin{aligned}
& \Phi=x_{1}^{3}+2 x_{2}^{3}+6 x_{3}^{3}-4 x_{4}^{3} \quad \equiv 0(\bmod 7), \\
& \Psi=\quad x_{2}^{3}+2 x_{3}^{3}+4 x_{4}^{3}+x_{5}^{3} \equiv 0(\bmod 7)
\end{aligned}
$$

have no non-trivial solutions. Adjoining a third congruence, say

$$
\Xi=x_{6}^{3}+x_{7}^{3}+x_{8}^{3} \equiv 0(\bmod 7),
$$

we can obtain a system of three congruences $(\bmod 7)$ which only have singular solutions. In theory, for the exceptional primes $p \geq 7$, it is possible to decide the question of $p$-adic solutions computationally. We outline a procedure for this in $\S \S 12-15$, although the computations are too long to be practical at present. We have not been able to settle the question of 3 -adic solutions; the indications from Davenport and Lewis [15] and Cook [12] are that the 3-adic case could take up more pages than all the other primes put together. For any given system of 3 equations it is reasonably straightforward to check if there are solutions in the exceptional $p$-adic fields not settled by the theorem.

2. Preliminaries. Let $\gamma=\gamma(p)$ be given by $\gamma(p)=1, p \neq 3, \gamma(3)=2$. Hensel's lemma shows that the equations (2.1) will have a non-trivial $p$-adic solution provided that the congruences

$$
F(\boldsymbol{x}) \equiv G(\boldsymbol{x}) \equiv H(\boldsymbol{x}) \equiv 0\left(\bmod p^{\gamma}\right)
$$


have a solution of rank 3. A solution $\boldsymbol{x}=\boldsymbol{\zeta}$ of the congruences (2.4) is said to be of rank $S$ if the matrix

$$
\left[\begin{array}{lll}
a_{1} \zeta_{1} & \ldots & a_{N} \zeta_{N} \\
b_{1} \zeta_{1} & \ldots & b_{N} \zeta_{N} \\
c_{1} \zeta_{1} & \ldots & c_{N} \zeta_{N}
\end{array}\right]
$$

has rank $S(\bmod p)$.

LEMma 13. Suppose that the congruences (2.4) have a solution of rank 3. Then the equations (2.1) have a non-trivial p-adic solution.

This is Lemma 9 of Davenport and Lewis [17].

In order to obtain a solution of rank 3 we use a $p$-adic normalization argument due to Davenport and Lewis [17]. Since it works in the general setting of $R$ additive equations of odd degree $k>1$,

$$
F_{i}(\boldsymbol{x})=a_{i 1} x_{1}^{k}+\ldots+a_{i N} x_{N}^{k}=0, \quad i=1, \ldots, R,
$$

we state the results in this more general setting.

Two systems of additive forms $F_{1}, \ldots, F_{R}$ and $G_{1}, \ldots, G_{R}$ are said to be $p$-equivalent if one system can be obtained from the other by a combination of the operations

$$
\text { (I) } \quad f_{i}^{\prime}(\boldsymbol{x})=f_{i}\left(p^{v(1)} x_{1}, \ldots, p^{v(N)} x_{N}\right) \quad \text { for } 1 \leq i \leq R,
$$

where $v(1), \ldots, v(N)$ are integers and

$$
\text { (II) } f_{i}^{\prime \prime}(\boldsymbol{x})=\alpha_{i 1} f_{1}(\boldsymbol{x})+\ldots+\alpha_{i R} f_{R}(\boldsymbol{x}) \quad \text { for } 1 \leq i \leq R,
$$

where the $\alpha_{i j}$ are rational numbers with $\operatorname{det}\left(\alpha_{i j}\right) \neq 0$. If the system $F_{1}, \ldots$ $\ldots, F_{R}$ has a simultaneous non-trivial zero in the $p$-adic field then so does any $p$-equivalent system.

Let

$$
\theta\left(F_{1}, \ldots, F_{R}\right)=\prod_{a} \operatorname{det}(a)
$$

where the product is taken over all $R \times R$ submatrices of $\left(a_{i j}\right)$. This definition differs slightly from that in $\S 4$ of Davenport and Lewis [17], where permutations of the columns of $a$ are allowed, the effect of which is to raise $\theta$ to the power of $R$ ! and does not affect the following arguments for $R \geq 3$. As in $\S 4$ of Davenport and Lewis [17] a $p$-adic compactness argument shows that it is sufficient to prove the theorem under the additional hypothesis that

$$
\theta\left(F_{1}, \ldots, F_{R}\right) \neq 0
$$

since if $\theta=0$ we can choose sequences of forms $F_{1}^{\mu}, \ldots, F_{R}^{\mu}$ converging to $F_{1}, \ldots, F_{R}$ p-adically and with $\theta\left(F_{1}^{\mu}, \ldots, F_{R}^{\mu}\right) \neq 0$. 
We may now assume that " $\theta \neq 0$ ", a property that is preserved under $p$-equivalence. From all the systems of forms that are $p$-equivalent to $F_{1}, \ldots, F_{R}$, and so have $\theta \neq 0$, and have integral coefficients we select a system $f_{1}, \ldots, f_{R}$ for which the power of $p$ dividing $\theta\left(f_{1}, \ldots, f_{R}\right)$ is least. Such a system of forms is said to be p-normalized.

LEMMA 14. A p-normalized system of forms can be written (after renumbering the variables) as

$$
f_{i}(\boldsymbol{x})=F_{i}^{*}\left(x_{1}, \ldots, x_{m}\right)+p G_{i}^{*}\left(x_{m+1}, \ldots, x_{N}\right)
$$

for $i=1, \ldots, R$ where

$$
m \geq N / k
$$

and each of $x_{1}, \ldots, x_{m}$ occurs in at least one of $F_{1}^{*}, \ldots, F_{R}^{*}$ with a coefficient not divisible by $p$.

Moreover, if we form any s linearly independent combinations of $f_{1}^{*}, \ldots$ $\ldots, f_{R}^{*}(\bmod p)$ and denote by $q_{s}$ the minimum number of variables occurring in at least one of these combinations with a coefficient not divisible by $p$ then

$$
q_{s} \geq s N / R k
$$

for $s=1, \ldots, R$.

This is Lemma 11 of Davenport and Lewis [17].

Returning now to the specific case $k=3, N \geq 22$ we have to show that the congruences

$$
\begin{aligned}
F^{*} & \equiv a_{1} x_{1}^{3}+\ldots+a_{m} x_{m}^{3} \equiv 0\left(\bmod p^{\gamma}\right) \\
G^{*} & \equiv b_{1} x_{1}^{3}+\ldots+b_{m} x_{m}^{3} \equiv 0\left(\bmod p^{\gamma}\right) \\
H^{*} & \equiv c_{1} x_{1}^{3}+\ldots+c_{m} x_{m}^{3} \equiv 0\left(\bmod p^{\gamma}\right)
\end{aligned}
$$

where

$$
m \geq 8, \quad q=q_{1} \geq 3 \quad \text { and } \quad q_{2} \geq 5,
$$

have a solution of rank $3(\bmod p)$.

Lemma 15. If $p \equiv 2(\bmod 3)$ then the congruences $(2.13)$ have a solution of rank $3(\bmod p)$.

P r o of. In this case every residue $(\bmod p)$ is a cubic residue, so that after a substitution $y_{i}=x_{i}^{3}$ we may treat the congruences as linear equations in $\boldsymbol{Z}_{p}$. Relabelling the variables and using row operations we may take the matrix of coefficients as

$$
\left[\begin{array}{ll}
I & \boldsymbol{c}_{4} \ldots \boldsymbol{c}_{m}
\end{array}\right]=\left[\begin{array}{ll}
I & C
\end{array}\right]
$$


say, where $I$ is the $3 \times 3$ identity matrix and $\boldsymbol{c}_{4}, \ldots, \boldsymbol{c}_{m}$ are the columns of $C$. We take $y_{1}=y_{2}=y_{3}=1$ and solve $C \boldsymbol{\eta}=-(1,1,1)^{T}$ to give a solution of rank 3 .

Lemma 16. The congruence

$$
a x^{3}+b y^{3}+c z^{3} \equiv 0(\bmod p)
$$

is always soluble with at least one of $x, y, z \not \equiv 0(\bmod p)$.

This is Theorem 1 of Lewis [19].

Let $r, s, t$ denote the lengths of the longest, second longest and third longest blocks of columns lying in a 1-dimensional subspace of $\boldsymbol{Z}_{p}^{3}$. Then $r \geq s \geq t \geq 1$. Let $\mu(d)$ denote the maximum number of columns lying in a $d$-dimensional subspace of $\boldsymbol{Z}_{p}^{3}$. Then

$$
q_{d}=m-\mu(3-d)
$$

in particular, $r=\mu(1)$ and $q_{2}=m-r$. Thus the inequalities

$$
m \geq 8, \quad q_{2} \geq 5, \quad q \geq 3
$$

are equivalent to

$$
m \geq 8, \quad \mu(1) \leq m-5, \quad \mu(2) \leq m-3 .
$$

We observe that if $\mu(1)=m-5, \mu(2)=m-3$ and the corresponding 1and 2-dimensional subspaces of columns are disjoint then

$$
m \geq \mu(1)+\mu(2)=2 m-8,
$$

or $m \leq 8$.

Lemma 17. Suppose that $m>8$. Then either we can choose a subset of 8 columns and still have $q \geq 3$ and $q_{2} \geq 5$, or there is a subset of 9 columns which can be partitioned into 3 independent 1-dimensional subsets, each containing 3 columns.

P r o of. While $m>8$ we reduce $m$ to $m-1$ using the following rule:

If $\mu(2)<m-3$ or if $\mu(2)=m-3$ and $\mu(1)=m-5$ we discard a column from the longest 1-dimensional block of columns. This reduces $m$ to $m-1$ and preserves the properties " $\mu(1) \leq m-5$ " and " $\mu(2) \leq m-3$ " since the blocks cannot be disjoint. (If there were 21 -dimensional blocks of columns of length $m-5$ then $\mu(2) \geq 2 m-10 \geq m-1$, contrary to (2.18).)

Otherwise we have $\mu(1)<m-5$ and $\mu(2)=m-3$. In this case we discard a column from the longest 2-dimensional block of columns. This reduces $m$ to $m-1$ and preserves the properties " $\mu(1) \leq m-5$ " and " $\mu(2) \leq m-3$ ". (If there were 2 disjoint 2-dimensional blocks of length $m-3$ then $m \geq 2(m-3)$, or $m \leq 6$; otherwise we discard a column in the intersection of the 2 blocks. Since $\mu(1)<m-5$, any 22 -dimensional blocks of length $m-3$ meet in exactly $m-6$ columns. Then the $m$ columns fall into 1-dimensional blocks 
of length 3,3 and $m-6$. If $m>9$ we discard a column from the third of these blocks.)

In the second of the possible outcomes described in Lemma 17 we may suppose that the independent columns are multiples of the orthonormal basis $\boldsymbol{e}_{1}, \boldsymbol{e}_{2}, \boldsymbol{e}_{3}$. The required solution of rank 3 then follows by applying Lemma 16 to 3 independent congruences. We shall now suppose that $p \equiv 1(\bmod 3)$, so that $\gamma=1$, and $m=8, q_{2} \geq 5$ and $q \geq 3$ in the congruences (2.13).

3. The case $r=3$. In this case the system of congruences (2.13) is equivalent to one with coefficient matrix

$$
\left[\begin{array}{cccccccc}
1 & \alpha_{1} & \alpha_{2} & 0 & 0 & a_{1} & a_{2} & a_{3} \\
0 & 0 & 0 & 1 & 0 & b_{1} & b_{2} & b_{3} \\
0 & 0 & 0 & 0 & 1 & c_{1} & c_{2} & c_{3}
\end{array}\right]
$$

where $\alpha_{1} \alpha_{2} \not \equiv 0(\bmod p)$.

Lemma 18. Let $p>7$. Suppose that

$$
f=a_{1} x_{1}^{3}+\ldots+a_{\nu} x_{\nu}^{3}, \quad g=b_{1} x_{1}^{3}+\ldots+b_{\nu} x_{\nu}^{3}
$$

where $\nu \geq 5$ and $a_{i}, b_{i}$ are not both zero $(\bmod p)$ for $i=1, \ldots, \nu$. Suppose further that any form $\lambda f+\mu g,(\lambda, \mu) \not \equiv(0,0)(\bmod p)$, contains at least 3 variables with non-zero coefficients $(\bmod p)$. Then the congruences

$$
f(\boldsymbol{x}) \equiv g(\boldsymbol{x}) \equiv 0(\bmod p)
$$

have a solution of rank $2(\bmod p)$.

This is proved in $\S \S 3-5$ of Cook [12].

Now $q \geq 3$ so we can find a solution $\zeta$ of the congruences

$$
\begin{aligned}
\zeta_{4}^{3}+b_{1} \zeta_{1}^{3}+b_{2} \zeta_{2}^{3}+b_{3} \zeta_{3}^{3} & \equiv 0(\bmod p), \\
\zeta_{5}^{3}+c_{1} \zeta_{1}^{3}+c_{2} \zeta_{2}^{3}+c_{3} \zeta_{3}^{3} & \equiv 0(\bmod p)
\end{aligned}
$$

having rank $2(\bmod p)$. In order to solve the congruences corresponding to (2.20) we now need to solve

$$
x_{1}^{3}+\alpha_{2} x_{2}^{3}+\alpha_{3} x_{3}^{3} \equiv d
$$

where $d=-a_{1} \zeta_{1}^{3}-a_{2} \zeta_{2}^{3}-a_{3} \zeta_{3}^{3}$. This we achieve with the following lemma, which is the case $k=3$ of Davenport and Lewis [14].

Lemma 19. The congruence

$$
a x^{3}+b y^{3}+c z^{3} \equiv d(\bmod p)
$$

is always soluble if abcd $\not \equiv 0(\bmod p)$.

(If $d \equiv 0(\bmod p)$ then the solubility follows from Lemma 16. )

The solution constructed in this way clearly has rank $3(\bmod p)$, so we have proved 
LEMma 20. Let $p>7$ and $p \equiv 1(\bmod 3)$. If $r \geq 3$ then the congruences (2.13) have a solution of rank $3(\bmod p)$.

4. The case $q=3$. In this case the congruences (2.13) are equivalent to a system with coefficient matrix

$$
\left[\begin{array}{cccccccc}
1 & 0 & 0 & a_{1} & a_{2} & a_{3} & a_{4} & a_{5} \\
0 & 1 & 0 & b_{1} & b_{2} & b_{3} & b_{4} & b_{5} \\
0 & 0 & 1 & c_{1} & c_{2} & 0 & 0 & 0
\end{array}\right]
$$

where $c_{1} c_{2} \not \equiv 0(\bmod p)$.

Lemma 21. For $p \equiv 1(\bmod 3), p>7$, the congruence

$$
a x^{3}+b y^{3} \equiv c(\bmod p)
$$

is always soluble if $a b c \not \equiv 0(\bmod p)$.

This is the case $k=3$ of Theorem 3 of Chowla, Mann and Straus [9].

Lemma 22. For $p \equiv 1(\bmod 3), p>7$, and $a b c \not \equiv 0(\bmod p)$ the congruence

$$
a x^{3}+b y^{3}+c z^{3} \equiv 0(\bmod p)
$$

is always soluble with $x y \not \equiv 0(\bmod p)$.

This is essentially Lemma 6 of Stevenson [21]; she assumed that $c=1$ but we can multiply by $c^{-1}(\bmod p)$ to reduce to that case.

We begin by solving the congruence

$$
z^{3}+c_{1} y_{1}^{3}+c_{2} y_{2}^{3} \equiv 0(\bmod p)
$$

with $z y_{1} \not \equiv 0(\bmod p)$. We then have to solve 2 congruences of the form

$$
\begin{aligned}
& f_{0}=x_{1}^{3}+a_{3} x_{3}^{3}+a_{4} x_{4}^{3}+a_{5} x_{5}^{3} \equiv A(\bmod p), \\
& g_{0}=x_{2}^{3}+b_{3} x_{3}^{3}+b_{4} x_{4}^{3}+b_{5} x_{5}^{3} \equiv B(\bmod p) .
\end{aligned}
$$

Lemma 23. Let $p \equiv 1(\bmod 3), p>7$. Suppose that any linear combination $\lambda f_{0}+\mu g_{0}$, where $(\lambda, \mu) \not \equiv(0,0)(\bmod p)$, contains at least $q^{*}$ variables explicitly $(\bmod p)$, where $q^{*} \geq 3$. Then the congruences $(2.26)$ have a solution of rank $2(\bmod p)$.

Proof. When $A \equiv B \equiv 0(\bmod p)$ this follows from Lemma 18 so now we suppose that $A$ and $B$ are not both zero $(\bmod p)$. If $q^{*}=4$ then any non-trivial solution has $\operatorname{rank} 2(\bmod p)$, and the result follows from Lemma 8 of Stevenson [21].

Now $q^{*}=3$, the longest block of columns in a 1-dimensional space has length 2 so any solution of $(2.26)$ with at least 3 variables non-zero $(\bmod p)$ will have rank 2 . We can replace the congruences (2.26) with an equivalent 
system

$$
\begin{aligned}
& f_{1}(\boldsymbol{x})=x_{1}^{3}+a_{2} x_{2}^{3} \quad+a_{4} x_{4}^{3}+a_{5} x_{5}^{3} \equiv a(\bmod p), \\
& g_{1}(\boldsymbol{x})=x_{3}^{3}+b_{4} x_{4}^{3}+b_{5} x_{5}^{3} \equiv b(\bmod p)
\end{aligned}
$$

where $a$ and $b$ are not both zero $(\bmod p)$.

Suppose first that $b \equiv 0(\bmod p)$. Using Lemma 22 we can solve $g\left(x_{3}, x_{4}, x_{5}\right) \equiv 0(\bmod p)$ with $x_{3} x_{4} \not \equiv 0(\bmod p)$. Then $\left(-x_{3},-x_{4},-x_{5}\right)$ is also a solution. Let $a^{\prime}=a_{4} x_{4}^{3}+a_{5} x_{5}^{3}$. If $a-a^{\prime}, a+a^{\prime}$ are both zero $(\bmod p)$ then $a \equiv 0(\bmod p)$, contrary to $b \equiv 0(\bmod p)$ and $a, b$ not both zero $(\bmod p)$. Therefore we can choose one of the solutions $\pm\left(x_{3}, x_{4}, x_{5}\right)$ so that $c=a \pm a^{\prime} \not \equiv 0(\bmod p)$. Using Lemma 21 we can then solve $x_{1}^{3}+a_{2} x_{2}^{3} \equiv c(\bmod p)$ to give the required solution of rank 2 .

Now $b \not \equiv 0(\bmod p)$ and we choose $x_{3}= \pm 1$ so that $b^{\prime}=b-x_{3}^{3} \not \equiv$ $0(\bmod p)$, and using Lemma 21 we can solve $b_{4} x_{4}^{3}+b_{5} x_{5}^{3} \equiv b^{\prime}(\bmod p)$ and one of $x_{4}, x_{5}$ is non-zero $(\bmod p)$. The congruence for $f_{1}$ becomes

$$
x_{1}^{3}+a_{2} x_{2}^{3} \equiv a^{\prime}(\bmod p) .
$$

If $a^{\prime} \not \equiv 0(\bmod p)$ we can again use Lemma 21 to give a solution with one of $x_{1}, x_{2}$ non-zero $(\bmod p)$, and the simultaneous solution has rank 2 .

Now $a^{\prime} \equiv 0$ in (2.28) and we take $x_{1}=x_{2}=0$ in our simultaneous solution. If $x_{i} \not \equiv 0$ (where $i=4$ or 5 ) the solution has rank 2 unless $a_{i} \equiv 0(\bmod p)$, in which case, renumbering so that $i=4$, the congruences become

$$
\begin{aligned}
x_{1}^{3}+a_{2} x_{2}^{3}+a_{5} x_{5}^{3} & \equiv a(\bmod p), \\
x_{3}^{3}+b_{4} x_{4}^{3}+b_{5} x_{5}^{3} & \equiv b(\bmod p),
\end{aligned}
$$

where none of the coefficients are zero $(\bmod p)$. Thus the values $a-a_{5}$, $a, a+a_{5}$ are distinct $(\bmod p)$, and so are $b-b_{5}, b, b+b_{5}$. Thus we can take $x_{5}$ to be one of $0, \pm 1$ so that $a-a_{5} x_{5}^{3}, b-b_{5} x_{5}^{3}$ are both non-zero $(\bmod p)$. We can then solve the resulting congruences using Lemma 21, to give a simultaneous solution of rank $2(\bmod p)$ and complete the proof of the lemma.

The solution to (2.26) of rank 2 then gives a simultaneous solution of rank 3 to the congruences with coefficient matrix (2.22), completing the argument when $q=3$.

5. The case $t=2$. We now have $r=s=t=2$ and $q \geq 4$ so the congruences are equivalent to a system

$$
\begin{aligned}
x_{1}^{3}+a x_{2}^{3} & +a_{1} w_{1}^{3}+a_{2} w_{2}^{3} \equiv 0(\bmod p), \\
y_{1}^{3}+b y_{2}^{3} w_{1}^{3}+b_{2} w_{2}^{3} & \equiv 0(\bmod p), \\
z_{1}^{3}+c z_{2}^{3}+c_{1} w_{1}^{3}+c_{2} w_{2}^{3} & \equiv 0(\bmod p)
\end{aligned}
$$


where the coefficients are all non-zero. We take $w_{1}=-1, w_{2}=0$ and then solve three independent congruences

$$
x_{1}^{3}+a x_{2}^{3} \equiv a_{1}, \quad y^{3}+b y_{2}^{3} \equiv b_{1}, \quad z^{3}+c z_{2}^{3} \equiv c_{1}(\bmod p)
$$

using Lemma 21. Clearly this solution has rank $3(\bmod p)$.

Now we may suppose that $t=1$.

6. The case $s=2$. Now $r=s=2, t=1$ and we begin by observing that the congruences (2.13) are equivalent to a system with coefficient matrix

$$
\left[\begin{array}{cccccccc}
1 & a & 0 & 0 & a_{0} & a_{1} & a_{2} & a_{3} \\
0 & 0 & 1 & b & b_{0} & b_{1} & b_{2} & b_{3} \\
0 & 0 & 0 & 0 & c_{0} & c_{1} & c_{2} & c_{3}
\end{array}\right] .
$$

The cubic residues $(\bmod p)$ form a subgroup of the nonzero residue classes. If $Q$ is a cubic non-residue $(\bmod p)$ then $S=\left\{1, Q, Q^{2}\right\}$ is a set of representatives from the 3 cosets. Using substitutions $x \rightarrow \alpha x$ we can bring the coefficients $c_{0}, c_{1}, c_{2}, c_{3}$ into the set $S$. Then 2 of them, $c_{0}$ and $c_{1}$ say, must be equal and we multiply the third congruence by $c_{0}^{-1}$ to make $c_{0}=c_{1}=1$. We then replace $f_{0}, g_{0}$ by $f_{0}-a_{0} h_{0}, g_{0}-b_{0} h_{0}$ to give a coefficient matrix

$$
\left[\begin{array}{cccccccc}
1 & a & 0 & 0 & 0 & a_{1} & a_{2} & a_{3} \\
0 & 0 & 1 & b & 0 & b_{1} & b_{2} & b_{3} \\
0 & 0 & 0 & 0 & 1 & 1 & c_{2} & c_{3}
\end{array}\right] .
$$

Since $t=1, a_{1}$ and $b_{1}$ are not both 0 . If $a_{1} b_{1} \not \equiv 0(\bmod p)$ we take the last 4 variables to be $1,-1,0,0$ and solve

$$
x_{1}^{3}+a x_{2}^{3} \equiv a_{1}, \quad y_{1}^{3}+b y_{2}^{3} \equiv b_{1}(\bmod p)
$$

to give the required solution of rank 3 .

Now we may suppose that exactly one of $a_{1}, b_{1}$ is $0(\bmod p)$, say $a_{1} \equiv$ $0(\bmod p)$. Then $a_{2} a_{3} \not \equiv 0(\bmod p)$ since $q \geq 4$. If $c_{2}=c_{3}$ we take the last 4 variables to be $1,-1,1,-1$ and then solve two congruences

$$
\begin{aligned}
x^{3}+a x_{1}^{3} & \equiv a_{3}-a_{2}(\bmod p), \\
y^{3}+b y_{1}^{3} & \equiv b_{3}-b_{2}+b_{1}(\bmod p),
\end{aligned}
$$

where possibly the solution is trivial. However, the non-zero variables are based on the columns

$$
\begin{array}{ccc}
0 & 0 & a_{2} \\
0 & b_{1} & b_{2} \\
1 & 1 & c_{2}
\end{array}
$$

where $a_{2} \not \equiv 0(\bmod p)$, and so the solution has rank $3(\bmod p)$.

Now $c_{2} \neq c_{3}$ and we relabel so that $c_{2} \neq 1$. Using Lemma 22 we see that the congruence $z^{3}+w_{1}^{3}+c_{2} w_{2}^{3} \not \equiv 0(\bmod p)$ has a solution with 
$w_{1} w_{2} \not \equiv 0(\bmod p)$. Since $c_{2}$ is a cubic non-residue we must also have $z \not \equiv 0(\bmod p)$. We take the last variable $w_{3}=0$ and then solve two congruences $x^{3}+a x_{1}^{3} \equiv A, y^{3}+b y_{1}^{3} \equiv B(\bmod p)$, and again these solutions may be trivial. Again, the non-zero variables are based on the columns $(2.30)$ and so the solution has $\operatorname{rank} 3(\bmod p)$.

7. The case $r=2, q=5$. Now $r=2, s=t=1$ and the system is equivalent to

$$
\begin{aligned}
& f_{0}=x^{3}+a x_{1}^{3} \quad+a_{1} w_{1}^{3}+\ldots+a_{4} w_{4}^{3} \equiv 0(\bmod p), \\
& g_{0}=y^{3}+b_{1} w_{1}^{3}+\ldots+b_{4} w_{4}^{3} \equiv 0(\bmod p) \text {, } \\
& h_{0}=\quad z^{3}+c_{1} w_{1}^{3}+\ldots+c_{4} w_{4}^{3} \equiv 0(\bmod p) .
\end{aligned}
$$

Since $q \geq 3$ it follows from Lemma 18 that the congruences $g_{0} \equiv h_{0} \equiv$ $0(\bmod p)$ have a solution of rank 2 , and in particular must contain at least 3 non-zero variables. Then $f_{0} \equiv 0(\bmod p)$ becomes

$$
x^{3}+a x_{1}^{3} \equiv A(\bmod p)
$$

and if $A \not \equiv 0(\bmod p)$ this has a non-trivial solution. The resulting solution contains non-zero variables on 3 columns of the form

$$
\begin{array}{ccc}
\alpha & \alpha_{1} & \alpha_{2} \\
0 & \beta_{1} & \beta_{2} \\
0 & \gamma_{1} & \gamma_{2}
\end{array}
$$

where $\alpha\left(\beta_{1} \gamma_{2}-\beta_{2} \gamma_{1}\right) \not \equiv 0(\bmod p)$. Therefore the solution has rank 3 $(\bmod p)$.

Now we may suppose that the solution of $g_{0} \equiv h_{0} \equiv 0$ has $A \equiv 0(\bmod p)$.

Lemma 24. Suppose that $q>\ell$. Then any solution to the congruences $f_{0} \equiv g_{0} \equiv h_{0} \equiv 0(\bmod p)$ with at least $m-\ell$ variables non-zero is necessarily of rank 3 .

Proof. Recall that $\mu(2)$, the maximum number of columns in a 2dimensional space, is $m-q$. Therefore, in this solution, the non-zero variables cannot lie in a block of columns restricted to a 2-dimensional space. Therefore the solution has rank 3 .

Now $r=2$ so $q=4$ or 5 .

Suppose $q=5$. If at least 4 of $y, z, w_{1}, \ldots, w_{4}$ are non-zero then the common solution has rank $3(\bmod p)$. Now suppose that only 3 of $y, z, w_{1}, \ldots, w_{4}$ are non-zero. The corresponding sections of $g_{0}, h_{0}$ are equivalent to a pair where the coefficient matrix is

$$
\left[\begin{array}{lll}
1 & 0 & b \\
0 & 1 & c
\end{array}\right]
$$


and $b, c$ are cubic residues $(\bmod p)$. If this solution has $A \equiv 0$ then the system of congruences has coefficient matrix

$$
\left[\begin{array}{cccccccc}
1 & a & 0 & 0 & 0 & a_{1} & a_{2} & a_{3} \\
0 & 0 & 1 & 0 & b & b_{1} & b_{2} & b_{3} \\
0 & 0 & 0 & 1 & c & c_{1} & c_{2} & c_{3}
\end{array}\right]
$$

Consider the sections

$$
\begin{aligned}
f^{*} & =x^{3}+a x_{1}^{3}+a_{1} w_{1}^{3}+a_{2} w_{2}^{3}+a_{3} w_{3}^{3} \equiv 0(\bmod p), \\
g^{*} & =b_{1} w_{1}^{3}+b_{2} w_{2}^{3}+b_{3} w_{3}^{3} \equiv 0(\bmod p) .
\end{aligned}
$$

Suppose that some form $\lambda f^{*}+\mu g^{*},(\lambda, \mu) \not \equiv(0,0)(\bmod p)$, has $q^{*} \leq 2$ variables explicitly $(\bmod p)$. Then the corresponding form $\lambda f+\mu g$ has at most 4 variables and this is not possible since $q=5$. Therefore we may apply Lemma 18 to obtain a solution of rank $2(\bmod p)$. In particular, some non-zero variable will have a non-zero coefficient in $f^{*}$.

The third congruence then becomes $z^{3}+c w^{3} \equiv C(\bmod p)$ and, since $c$ is a cubic residue $(\bmod p)$, we can solve this with $w \not \equiv 0$ and then solve $y^{3} \equiv B=-b w^{3}(\bmod p)$. The non-zero variables are based on columns

$$
\begin{array}{lll}
0 & 0 & a^{\prime} \\
1 & b & b^{\prime} \\
0 & c & c^{\prime}
\end{array}
$$

where $a^{\prime} \not \equiv 0(\bmod p)$, and so the solution has rank 3 .

8. The case $r=2, q=4$. We begin as in $\S 7$. If at least 5 of $y, z, w_{1}, \ldots, w_{4}$ in our solution of $g_{0} \equiv h_{0} \equiv 0(\bmod p)$ are non-zero then we have a common solution of rank 3 .

Now suppose that exactly 4 of the variables are non-zero and that there is no solution with exactly 3 variables non-zero. Replacing $f$ by $f-a_{i} b_{i}^{-1} g$ etc. we may suppose that the non-zero variables are $y, z, w_{1}, w_{2}$. If the resulting solution of (2.31) has rank 2 then the coefficient matrix is

$$
\left[\begin{array}{cccccccc}
1 & a & 0 & 0 & 0 & 0 & a_{3} & a_{4} \\
0 & 0 & 1 & 0 & b_{1} & b_{2} & b_{3} & b_{4} \\
0 & 0 & 0 & 1 & c_{1} & c_{2} & c_{3} & c_{4}
\end{array}\right] .
$$

Since $s=1, b_{1} c_{2}-b_{2} c_{1} \not \equiv 0(\bmod p)$. Taking $w_{4}=-1$ we use Lemma 23 to give a solution of rank 2 to the congruences

$$
\begin{aligned}
y^{3}+b_{1} w_{1}^{3}+b_{2} w_{2}^{3}+b_{3} w_{3}^{3} & \equiv b_{4}(\bmod p), \\
z^{3}+c_{1} w_{1}^{3}+c_{2} w_{2}^{3}+c_{3} w_{3}^{3} & \equiv c_{4}(\bmod p) .
\end{aligned}
$$

Let $A=-a_{3} w_{3}^{3}+a_{4}$ and solve $x^{3}+a x_{1}^{3} \equiv A(\bmod p)$. If $A \not \equiv 0$ this gives the required solution of rank $3(\bmod p)$.

If $A \equiv 0$ then $w_{3} \not \equiv 0(\bmod p)$. Since we are assuming that there is no solution to $g_{0} \equiv h_{0}$ with just 3 variables non-zero at least 2 of $y, z, w_{1}, w_{2}$ 
are non-zero. If $w_{1} w_{2} \not \equiv 0$ we consider the variables $w_{1}, w_{2}, w_{3}$. If $z \not \equiv 0$ consider the variables $z, w_{3}$ and one of $y, w_{1}, w_{2}$. In each case the variables are non-zero and the corresponding columns have non-zero determinant. Now $z$ and one of $w_{1}$ and $w_{2}, w_{2}$ say, are zero $(\bmod p)$ so we consider the variables $y, w_{1}$, and $w_{3}$. Since $s=1$ we have $c_{1} \not \equiv 0$ so

$$
\left|\begin{array}{ccc}
0 & 0 & a_{3} \\
1 & b_{1} & b_{3} \\
0 & c_{1} & c_{3}
\end{array}\right|=a_{3} c_{1} \not \equiv 0
$$

$\left(a_{3} \not \equiv 0\right.$ because $\left.q=4\right)$. Thus we have a solution of $\operatorname{rank} 3(\bmod p)$.

Now suppose there is a solution of $g_{0} \equiv h_{0} \equiv 0$ with exactly 3 of $y, z, w_{1}, \ldots, w_{4}$ non-zero. We proceed as for the case $q=5$ and obtain the forms $f^{*}, g^{*}$ as in (2.33). If $q^{*} \geq 3$ then the proof goes through as in the case $q=5$ so now suppose that $q^{*} \leq 2$. Either

(a) one of the $b_{i}$ 's, $b_{1}$ say, is 0 and the coefficients in (2.33) are

$$
\begin{array}{ccccc}
1 & a & a_{1} & a_{2} & a_{3} \\
0 & 0 & 0 & b_{2} & b_{3} .
\end{array}
$$

We take $w_{2}=w_{3}=0$, solve

$$
x^{3}+a x_{1}^{3}+a_{1} w_{1}^{3} \equiv 0(\bmod p)
$$

non-trivially and then continue exactly as in the case $q=5$. Or

(b) the last 3 columns in (2.33) lie in a 1-dimensional space, $a_{i}=\lambda b_{i}$ for $i=1,2,3$. Then the system of congruences is equivalent to a system with coefficient matrix

$$
\left[\begin{array}{cccccccc}
1 & a & \alpha & 0 & \alpha_{1} & 0 & 0 & 0 \\
0 & 0 & 1 & 0 & b & b_{1} & b_{2} & b_{3} \\
0 & 0 & 0 & 1 & c & c_{1} & c_{2} & c_{3}
\end{array}\right] .
$$

Since $s=1$ we have $b_{i} \not \equiv 0$ and $b_{i} c_{j}-b_{j} c_{i} \not \equiv 0$ for $i \neq j, 1 \leq i, j \leq 3$. Now $b$ and $c$ are cubic residues so we can solve

$$
\begin{aligned}
y^{3}+b w^{3} & \equiv 0(\bmod p), \\
z^{3}+c w^{3} & \equiv 0(\bmod p)
\end{aligned}
$$

with all 3 variables non-zero. Let $A=-\alpha y^{3}-\alpha_{1} w^{3}$. If $A \not \equiv 0$ we use a non-trivial solution of $x^{3}+\alpha_{1} x_{1}^{3} \equiv A$ to give a solution of rank 3 . If $A \equiv 0$ the solution $(y, z, w)$ still gives a solution of rank $3(\bmod p)$ unless $\alpha_{1} \equiv \alpha b$.

So far we have used $S=\left\{1, Q, Q^{2}\right\}$ as our set of coset representatives, however Davenport and Lewis [15] showed (Lemma 9) that there is a set $S_{1}=\{1, A, B\}$ of coset representatives with

$$
1+A+B \equiv 0(\bmod p) .
$$

Using substitutions $x \rightarrow \alpha x$ we can bring the coefficients $c, c_{1}, c_{2}, c_{3}$ into $S_{1}$. Since $c$ is a cubic residue it will be 1 . Either $c_{1}, c_{2}, c_{3}$ contain a 
repetition, say $c_{1}=c_{2}$, or $c_{1}, c_{2}, c_{3}$ are $1, A, B$. We construct a solution of $h_{0} \equiv 0$ by taking $z=-w=u$ and either $w_{1}=-w_{2}=v, w_{3}=0$ if $c_{1}=c_{2}$, or $w_{1}=w_{2}=w_{3}=v$ if $c_{1}, c_{2}, c_{3}$ are $1, A, B$. The congruences $f_{0} \equiv g_{0} \equiv 0$ become

$$
\begin{aligned}
x^{3}+a x_{1}^{3}+\alpha y^{3}-\alpha b u^{3} & \equiv 0(\bmod p), \\
y^{3}-b u^{3}+\beta v^{3} & \equiv 0(\bmod p) .
\end{aligned}
$$

If $\beta \equiv 0(\bmod p)$ we solve $y^{3} \equiv b u^{3}$ to give a solution of rank 3 . Otherwise we can apply Lemma 18 to give a solution of rank 2 to these congruences (2.34), and this gives a solution of rank 3 to the congruences (2.31).

From now on we may suppose that $r=1$.

9. The case $q=6$. Now the columns of coefficients lie in general position in $\boldsymbol{Z}_{p}^{3}$, so any non-trivial solution of $f_{0} \equiv g_{0} \equiv h_{0}(\bmod p)$ has rank 3. We use exponential sums to count the solutions. The number $N$ of solutions $(\bmod p)$ to the congruences $(2.13)$ is given by

$$
p^{3} N=\sum_{u_{1}, u_{2}, u_{3}} \sum_{(\bmod p)} T\left(\Lambda_{1}\right) \ldots T\left(\Lambda_{8}\right),
$$

where

$$
\begin{aligned}
\Lambda_{j} & =u_{1} a_{j}+u_{2} b_{j}+u_{3} c_{j}, \\
T(\Lambda) & =\sum_{x(\bmod p)} e\left(\Lambda x^{3} / p\right) .
\end{aligned}
$$

Separating out the term $u_{1}=u_{2}=u_{3}=0$ in (2.35) we see that

$$
p^{3} N-p^{8}=\sum \sum_{\boldsymbol{u} \neq \mathbf{0}} \sum T\left(\Lambda_{1}\right) \ldots T\left(\Lambda_{8}\right) .
$$

We classify the points $\boldsymbol{u} \not \equiv \mathbf{0}$ according to the number $\tau$ of linear forms $\Lambda_{i}$ which are $0(\bmod p)$. Since $q=6$ we have $\tau \leq 2$ and

$$
p^{3} N-p^{8}=\sum_{0}+\sum_{1}+\sum_{2}
$$

where $\sum_{j}$ is the sum over those points $\boldsymbol{u}(\bmod p)$ for which exactly $j$ forms $\Lambda_{i}$ are $0(\bmod p)$.

Since any 3 different forms $\Lambda_{i}, \Lambda_{j}, \Lambda_{k}$ are independent, the mapping $\left(\Lambda_{i}, \Lambda_{j}, \Lambda_{k}\right) \rightarrow\left(u_{1}, u_{2}, u_{3}\right)$ is a bijection $(\bmod p)$ and so

$$
\begin{aligned}
\left|\sum_{0}\right| & \leq\left.\max _{\substack{i, j, k \\
\text { distinct }}} \sum_{\Lambda_{i} \neq 0, \Lambda_{j} \neq 0, \Lambda_{k} \neq 0} \sum_{u_{1} \neq 0, u_{2} \neq 0, u_{3} \neq 0}\left|T\left(\Lambda_{i}\right) T\left(\Lambda_{j}\right) T\left(\Lambda_{k}\right)\right|^{8 / 3} T\left(u_{2}\right) T\left(u_{3}\right)\right|^{8 / 3}=\left(\sum_{u \neq 0}|T(u)|^{8 / 3}\right)^{3} . \\
& =\sum_{u_{1}} \sum \sum_{i} \mid T\left(u_{1}\right)
\end{aligned}
$$


Let

$$
S_{r}=\sum_{u \neq 0}|T(u)|^{r} .
$$

We have, from Lemma 2.5.1 of Dodson [18],

$$
S_{2}=2 p(p-1)
$$

and, from eq. (35) of Cook [12],

$$
S_{4}=6 p^{2}(p-1) .
$$

From Hölder's inequality we have

$$
S_{8 / 3} \leq S_{2}^{2 / 3} S_{4}^{1 / 3}
$$

and so

$$
\left|\sum_{0}\right| \leq S_{2}^{2} S_{4}=24 p^{4}(p-1)^{3} .
$$

The contribution to $\sum_{1}$ coming from those points $\boldsymbol{u} \not \equiv \mathbf{0}$ for which $\Lambda_{8} \equiv 0$ is at most

$$
p \sum_{\boldsymbol{u} \neq \mathbf{0}, \Lambda_{8} \equiv 0} \sum_{i}\left|T\left(\Lambda_{1}\right) \ldots T\left(\Lambda_{7}\right)\right| \leq p \max _{\substack{i \neq j \\ i \neq 8}} \sum_{\substack{\boldsymbol{u} \neq \mathbf{0}, \Lambda_{8} \equiv 0 \\ \Lambda_{i} \neq 0, \Lambda_{j} \neq 0}} \sum_{j}\left|T\left(\Lambda_{i}\right) T\left(\Lambda_{j}\right)\right|^{7 / 2} .
$$

The mapping $\left(\Lambda_{i}, \Lambda_{j}, \Lambda_{8}\right) \rightarrow\left(u_{1}, u_{2}, u_{3}\right)$ is a bijection, so the right hand term is

$$
p \sum_{u_{1} \neq 0} \sum_{u_{2} \neq 0}\left|T\left(u_{1}\right) T\left(u_{2}\right)\right|^{7 / 2}=p\left(\sum_{u \neq 0}|T(u)|^{7 / 2}\right)^{2} .
$$

From Hölder's inequality we have $S_{7 / 2} \leq S_{2}^{1 / 4} S_{4}^{3 / 4}$ so

$$
\left|\sum_{1}\right| \leq 8 p S_{2}^{1 / 2} S_{4}^{3 / 2}=96 \sqrt{3} p^{9 / 2}(p-1)^{2} .
$$

The contribution to $\sum_{2}$ coming from those points $\boldsymbol{u} \not \equiv \mathbf{0}$ for which $\Lambda_{7} \equiv \Lambda_{8} \equiv 0$ is at most

$$
\begin{aligned}
& p^{2} \sum_{\boldsymbol{u} \neq \mathbf{0}, \sum_{\Lambda_{7}} \sum_{\Lambda_{8} \equiv 0}\left|T\left(\Lambda_{1}\right) \ldots T\left(\Lambda_{6}\right)\right|} \\
& \quad \leq p^{2} \max _{i} \sum_{\Lambda_{7} \equiv \Lambda_{8} \equiv 0, \Lambda_{i} \neq 0}\left|T\left(\Lambda_{i}\right)\right|^{6}=p^{2} \sum_{u \neq 0}|T(u)|^{6}=p^{2} S_{6},
\end{aligned}
$$

since the mapping $\left(\Lambda_{i}, \Lambda_{7}, \Lambda_{8}\right) \rightarrow\left(u_{1}, u_{2}, u_{3}\right)$ is a bijection. Thus

$$
\left|\sum_{2}\right| \leq\left(\begin{array}{l}
8 \\
2
\end{array}\right) p^{2} S_{6}=28 p^{2} S_{6}
$$

Now for $u \not \equiv 0(\bmod p)$ we have

$$
|T(u)| \leq 2 \sqrt{p}
$$




$$
S_{6} \leq 4 p S_{4}=24 p^{3}(p-1) .
$$

From (2.39) we now deduce

$$
\begin{aligned}
N & \geq p^{5}-p^{-3}\left(\sum_{0}+\sum_{1}+\sum_{2}\right) \\
& \geq p^{5}-p^{-3}\left(24 p^{7}+96 \sqrt{3} p^{13 / 2}+672 p^{6}\right) \\
& =p^{3}\left\{p^{2}-(24 p+96 \sqrt{3 p}+672)\right\}>1
\end{aligned}
$$

for $p>60$.

10. The case $q=5$. We can suppose that the form $h_{0}$ contains 5 non-zero coefficients and that these coefficients are in the set $S=\{1, A, B\}$ of coset representatives with $1+A+B \equiv 0(\bmod p)$. We may also suppose that the coefficients $c_{0}, \ldots, c_{4}$, say, satisfy $c_{0}=c_{1}=1$ and either $c_{2}=c_{3}$ (type I) or $c_{2}=1, c_{3}=A, c_{4}=B$ (type II). Now the coefficient matrix can be taken as

$$
\left[\begin{array}{cccccccc}
1 & 0 & 0 & a & a_{1} & \ldots & a_{4} \\
0 & 1 & 0 & b & b_{1} & \ldots & b_{4} \\
0 & 0 & 1 & 0 & 1 & c_{2} & c_{3} & c_{4}
\end{array}\right] .
$$

We solve $h_{0} \equiv 0$ by taking $-z=w_{1}=\zeta$ and either $w_{2}=-w_{3}=\eta$ (type $\mathrm{I}$ ) or $w_{2}=w_{3}=w_{4}=\eta$ (type II). The first two congruences then become

$$
\begin{aligned}
x^{3}+a w^{3}+a_{1} \zeta^{3}+\alpha \eta^{3} & \equiv 0(\bmod p), \\
y^{3}+b w^{3}+b_{1} \zeta^{3}+\beta \eta^{3} & \equiv 0(\bmod p) .
\end{aligned}
$$

Suppose that $\alpha \equiv \beta \equiv 0(\bmod p)$. If the coefficients are of type I then $a_{2}=a_{3}, b_{2}=b_{3}, c_{2}=c_{3}$, the two columns are equal and $r>1$. Now the coefficients are of type II, $c_{2}=1$ and we have

$$
\begin{aligned}
a_{2}+a_{3}+a_{4} & \equiv 0(\bmod p), \\
b_{2}+b_{3}+b_{4} & \equiv 0(\bmod p), \\
1+c_{3}+c_{4} & \equiv 0(\bmod p) .
\end{aligned}
$$

Since we also have $c_{1}=1$ we replace the column with index 2 by the column with index 1 . If the congruences (2.54) still hold then the two columns are equal and $r>1$.

Lemma 25. Let $p>13, p \equiv 1(\bmod 3)$. If $a b c \not \equiv 0(\bmod p)$ then the congruence

$$
a x^{3}+b y^{3}+c z^{3} \equiv 0(\bmod p)
$$

has a solution with $x y z \not \equiv 0(\bmod p)$.

Proof. Using substitutions $x \rightarrow \alpha x$ etc. we may suppose that the coefficients $a, b, c \in S=\left\{1, Q, Q^{2}\right\}$. If $a, b, c$ are not all equal $(\bmod p)$ we 
may suppose that $b \neq c$. By Lemma 22 there is a solution with $y z \not \equiv 0$. Since $b \neq c$ we also have $x \not \equiv 0$.

Now $a, b, c$ are equal, on multiplying by $a^{-1}$ the congruence is

$$
x^{3}+y^{3}+z^{3} \equiv 0(\bmod p)
$$

and we count the number $N$ of solutions $(\bmod p)$, using exponential sums. We have

$$
\left|N-p^{2}\right| \leq p^{-1} S_{3} \leq p^{-1} 2 \sqrt{p} S_{2}=4 p^{1 / 2}(p-1) .
$$

The number of solutions to (2.56) with exactly 2 variables non-zero is $9(p-1)$, and there is one trivial solution. Thus we have a solution with $x y z \not \equiv 0(\bmod p)$ provided that

$$
p^{2}>4 p^{1 / 2}(p-1)+9(p-1)+1,
$$

and this is satisfied when $p \geq 37$. When $p=19$ we take $x=1, y=-2$ and $z=4$, and when $p=31$ we take $x=-1, y=8, z=8$.

Now we return to the congruences (2.53) and suppose that $a_{1} \equiv \alpha \equiv 0$. We take $x=w=0$ and solve

$$
y^{3}+b_{1} \zeta^{3}+\beta \eta^{3} \equiv 0
$$

with all three variables non-zero, then take $z=-\zeta$. The resulting solution to the system of 3 congruences has at least 5 variables non-zero and so has rank 3, from Lemma 24. A similar argument works if $b_{1} \equiv \beta \equiv 0(\bmod p)$.

Now suppose that " $q^{*} \leq 2$ " for the congruences (2.53), i.e. three of the columns lie in a 1-dimensional subspace. The only remaining possibility is that $a_{1} / b_{1} \equiv a_{2} / b_{2} \equiv \alpha / \beta(\bmod p)$. We solve the single congruence

$$
a w^{3}+a_{1} \zeta^{3}+\alpha \eta^{3} \equiv 0(\bmod p)
$$

with all three variables non-zero, and then take $z=-\zeta$. This provides a solution to the system of congruences with at least 5 variables non-zero, and so of rank 3 .

Now " $q^{*} \geq 3$ " so we can apply Lemma 19 to show that the congruences (2.53) have a solution of rank 2 , and so with at least 3 variables non-zero. If $\zeta \eta \not \equiv 0$ we have $z, w_{1}, w_{2}$ and $w_{3}$ non-zero, and since $q=5$ Lemma 24 shows that the solution has rank 3 .

Suppose that $\eta \equiv 0$ but $\zeta \not \equiv 0$. We have $z=-\zeta \not \equiv 0$ and two of $x, y, w$ are non-zero. Thus we have a solution supported by three columns

$$
\begin{array}{ccc}
0 & \alpha^{*} & \alpha^{\prime} \\
0 & \beta^{*} & \beta^{\prime} \\
1 & \gamma & \gamma
\end{array}
$$

where $\alpha^{*} \beta^{\prime}-\alpha^{\prime} \beta^{*} \not \equiv 0$, and so the solution has rank $3(\bmod p)$. 
Suppose that $\zeta \equiv 0$ but $\eta \not \equiv 0$. Then 2 of $x, y, w$ are non-zero so we have a solution supported by columns $\left[a_{i}, b_{i}, c_{i}\right]^{T}$ for some $i$ and two of

$\begin{array}{lll}1 & 0 & a \\ 0 & 1 & b \\ 0 & 0 & 0\end{array}$

where $a b \not \equiv 0(\bmod p)$. Therefore the solution has rank 3 .

We are now left with the case when the only solutions of (2.53) have $\zeta \equiv \eta \equiv 0(\bmod p)$. We have a solution to

$$
\begin{aligned}
x^{3}+a w^{3} & \equiv 0(\bmod p), \\
y^{3}+b w^{3} & \equiv 0(\bmod p)
\end{aligned}
$$

with all three variables non-zero. The coefficients $a, b$ must be cubic residues $(\bmod p)$ and the number of non-trivial solutions $(\bmod p)$ to the congruences $(2.61)$ is $9(p-1)$. We repeat the calculations of $\S 9$, counting the number of solutions to the three congruences which have some variable other than $x, y, w$ non-zero.

We estimate $\sum_{3}$, the contribution coming from the terms with exactly 4 forms $\Lambda_{i} \equiv 0$ by noting that such $\Lambda_{i}$ lie in a 2-dimensional subspace. Since $r=1$ any two forms $\Lambda_{i}, \Lambda_{j}$ with $i \neq j$ are linearly independent. Since $q=5$ it follows that any two triples of forms of rank 2 can intersect in at most one form $\Lambda$. In order to estimate the number of such triples we identify the forms $\Lambda_{i}$ with the vertices of the complete graph $K_{8}$. A triple $\left(\Lambda_{i}, \Lambda_{j}, \Lambda_{k}\right)$ corresponds to a triangle $(i, j, k)$ in $K_{8}$. Since two triples can meet in at most one form the corresponding triangles are edge-disjoint. Each vertex of $K_{8}$ has degree 7 and so can lie in at most 3 such triangles, and therefore there are at most 8 such triangles in all. Hence

$$
\left|\sum_{3}\right| \leq 8 p^{3} S_{5}
$$

and

$$
\begin{aligned}
N & \geq p^{5}-p^{-3}\left(\sum_{0}+\sum_{1}+\sum_{2}+\sum_{3}\right) \\
& \geq p^{5}-p^{-3}\left(S_{8 / 3}^{3}+8 p S_{7 / 2}^{2}+28 p^{2} S_{6}+8 p^{3} S_{5}\right)>1+9(p-1)
\end{aligned}
$$

for $p>61$. However, the argument can be refined further. Suppose $(1,2,3)$ is a triple occurring in the term $\sum_{3}$. Then the forms $\Lambda_{1}, \Lambda_{2}, \Lambda_{3}$ lie in a 2 dimensional space, and any two of them are linearly independent. Therefore the pairs $(1,2),(2,3)$ and $(3,1)$ cannot occur in the contribution $\sum_{2}$. Thus the contribution $\sum_{2}+\sum_{3}$ can be bounded by

$$
\max _{0 \leq r \leq 8}\left(r p^{3} S_{5}+(28-3 r) p^{2} S_{6}\right)
$$

and we then deduce that $N>1+9(p-1)$ for $p=61$. 

be

11. The case $q=4$. In this case we can take the coefficient matrix to

$$
\left[\begin{array}{cccccccc}
1 & 0 & \alpha_{1} & \alpha_{2} & 0 & a_{1} & a_{2} & a_{3} \\
0 & 1 & \beta_{1} & \beta_{2} & 0 & b_{1} & b_{2} & b_{3} \\
0 & 0 & 0 & 0 & 1 & 1 & c_{2} & c_{3}
\end{array}\right] .
$$

Since $r=1$ the coefficients $\alpha_{i}, \beta_{i}$ are non-zero and $\alpha_{1} \beta_{2}-\alpha_{2} \beta_{1} \not \equiv 0(\bmod p)$. Further, at least one of $a_{1}$ and $b_{1}$ is non-zero.

We begin by taking $-z=w_{1}=\zeta, w_{2}=w_{3}=0$ to give a solution to $h_{0} \equiv 0$. We then have to solve two congruences

$$
\begin{aligned}
x^{3}+\alpha_{1} v_{1}^{3}+\alpha_{2} v_{2}^{3}+a_{1} \zeta^{3} & \equiv 0, \\
y^{3}+\beta_{1} v_{1}^{3}+\beta_{2} v_{2}^{3}+b_{1} \zeta^{3} & \equiv 0,
\end{aligned}
$$

and we may apply Lemma 20 to give a solution of $\operatorname{rank} 2$. If $\zeta \not \equiv 0$ then this leads to a solution of rank 3 to the 3 simultaneous congruences.

We show that the congruences (2.63) have a solution with $\zeta \not \equiv 0$ by using exponential sums to count the number of solutions. The number $N_{0}$ of solutions to $(2.63)$ with $\zeta \equiv 0$ satisfies

$$
\begin{aligned}
\left|N_{0}-p^{2}\right| & \leq p^{-2} \sum_{\left(u_{1}, u_{2}\right)} \sum_{\neq(0,0)}\left|T\left(u_{1}\right) T\left(u_{2}\right) T\left(\Lambda_{3}\right) T\left(\Lambda_{4}\right)\right| \\
& =\left(p^{-1} S_{2}\right)^{2}=4(p-1)^{2}
\end{aligned}
$$

so

$$
N_{0} \leq p^{2}+4(p-1)^{2} .
$$

Suppose first that the columns of coefficients in (2.63) are pairwise linearly independent. Then the number $N$ of solutions to (2.63) satisfies

$$
\begin{aligned}
\left|N-p^{3}\right| & \leq p^{-2}\left(S_{5 / 2}^{2}+5 p S_{4}\right) \leq p^{-2}\left(S_{2}^{3 / 2} S_{4}^{1 / 2}+5 p S_{4}\right) \\
& =(48 p)^{1 / 2}(p-1)^{2}+30 p(p-1),
\end{aligned}
$$

using minor modifications to the arguments of Cook [12]. If the columns are not pairwise linearly independent then $a_{1} / b_{1}$ equals one of the other ratios $(\bmod p)$. There is now a contribution $\sum_{2}$ arising from points $\left(u_{1}, u_{2}\right)$ with two forms $\Lambda \equiv 0$, but there are only 3 forms $\Lambda_{i}$ for which exactly one $\Lambda \equiv 0$. Thus the upper bound in (2.65) can be replaced by

$$
\begin{array}{r}
p^{-2}\left(S_{5 / 2}^{2}+3 p S_{4}+p^{2} S_{3}\right) \leq p^{-2}\left(S_{2}^{3 / 2} S_{4}^{1 / 2}+3 p S_{4}+p^{2} S_{2}^{1 / 2} S_{4}^{1 / 2}\right) \\
=(48 p)^{1 / 2}(p-1)^{2}+18 p(p-1)+(12 p)^{1 / 2} p(p-1) .
\end{array}
$$

Thus we will have the required solution with $\zeta \not \equiv 0$ provided that $p^{3}-\left\{(48 p)^{1 / 2}(p-1)^{2}+(12 p)^{1 / 2} p(p-1)+18 p(p-1)\right\}>p^{2}+4(p-1)^{2}$, i.e. $p \geq 151$. 
For $p<151$ we repeat the calculations to see if

$$
p^{3}-p^{-2}\left(S_{5 / 2}^{2}+3 p S_{4}+p^{2} S_{3}\right)>p^{2}+4(p-1)^{2}
$$

and find that the required solution with $\zeta \not \equiv 0$ also exists for $p=151$ and 139.

12. The case $q=4$ continued. Now let $p<139$ be a fixed prime, $p \equiv 1(\bmod 3), p>13$. We consider the congruences $(2.63)$. If we can show that they have a solution with all variables non-zero $(\bmod p)$ then the arguments of $\S 11$ show that we have the desired solution of rank 3 .

Either $a_{1} \not \equiv 0$ or $b_{1} \not \equiv 0$ so we may suppose that $\beta_{1}, \beta_{2}, b_{1}$ are non-zero $(\bmod p)$. Let $Q$ be a cubic non-residue $(\bmod p)$ and $S=\left\{1, Q, Q^{2}\right\}$. Using substitutions $x \rightarrow \alpha x$ we have $\beta_{1}, \beta_{2}, b_{1} \in S$ and amongst the 4 coefficients of the second congruence, two will be equal. Thus we may replace the congruences (2.63) by

$$
\begin{aligned}
& f_{0}=x_{1}^{3}+a_{3} x_{3}^{3}+a_{4} x_{4}^{3}+a_{5} x_{5}^{3} \equiv 0(\bmod p), \\
& g_{0}=x_{2}^{3}+x_{3}^{3}+b_{4} x_{4}^{3}+b_{5} x_{5}^{3} \equiv 0(\bmod p)
\end{aligned}
$$

where $b_{3}, b_{4} \in S$. At most two of the columns can be linearly dependent and we suppose that these columns are multiples of $0 / 1$. Thus at most one of $a_{3}, a_{4}, a_{5}$ can be zero $(\bmod p)$ and $a_{i} / b_{i} \not \equiv a_{j} / b_{j}$ for $i \neq j(3 \leq i, j \leq 5)$.

Suppose first that $b_{4}=b_{5}$. Then

$$
\boldsymbol{x}=\left(x, u_{1},-u, v,-v\right)
$$

clearly satisfies $g_{0}(\boldsymbol{x}) \equiv 0(\bmod p)$. Further,

$$
f_{0}(\boldsymbol{x})=x^{3}-a_{3} u^{3}+\left(a_{4}-a_{5}\right) v^{3} .
$$

Since $b_{4}=b_{5}$ we have $a_{4}-a_{5} \not \equiv 0(\bmod p)$. Therefore, by Lemma 25 , the congruence $f_{0}(\boldsymbol{x}) \equiv 0$ has a solution with all variables non-zero $(\bmod p)$, provided that $p>13$.

Now we may assume that $b_{4} \neq b_{5}$ and see that there are only 3 cases to consider for $g_{0}$ (after renumbering $x_{4}$ and $x_{5}$ ):

$$
\begin{aligned}
& x_{2}^{3}+x_{3}^{3}+x_{4}^{3}+Q x_{5}^{3}, \\
& x_{2}^{3}+x_{3}^{3}+x_{4}^{3}+Q^{2} x_{5}^{3}, \\
& x_{2}^{3}+x_{3}^{3}+Q x_{4}^{3}+Q^{2} x_{5}^{3} .
\end{aligned}
$$

For each of these three cases $g_{0}$ we form a list of the solutions to $g_{0} \equiv$ $0(\bmod p)$ with all 4 variables non-zero (the list either contains all the solutions, or 1000 solutions if there are more). We then let $f_{0}$ run through all the possible forms by letting $a_{3}, a_{4}, a_{5}$ vary so that at most one $a_{i} \equiv 0$ and the ratios $a_{i} / b_{i}$ are distinct $(\bmod p)$. For each $f_{0}$ we run down the list of solutions to find a solution to $f_{0} \equiv 0(\bmod p)$.

(In fact the result still holds if we only have solutions with $x_{1}=0$, for 
we have $x_{2}, \ldots, x_{5}$ and $z$ non-zero. By Lemma 24 this will be enough to ensure that we have a solution of rank 3.)

In this way a computer verified that the congruences (2.67) have a solution with all variables non-zero, provided that $p \geq 31$.

13. The case $q=6$, computations. Now suppose $p>7$ is one of the remaining exceptional primes and begin by writing the coefficient matrix in the form

$$
\left[\begin{array}{cccccc}
1 & 0 & a & a_{1} & \ldots & a_{5} \\
0 & 1 & b & b_{1} & \ldots & b_{5} \\
0 & 0 & c & c_{1} & \ldots & c_{5}
\end{array}\right] .
$$

Let $S=\{1, A, B\}$ be a set of coset representatives $(\bmod p)$ for which $1+A+$ $B \equiv 0(\bmod p)$. Using substitutions $x \rightarrow \alpha x$ we can bring the coefficients $c, c_{1}, \ldots, c_{5}$ into $S$. There must be at least 2 pairs of repeated values amongst $c, c_{1}, \ldots, c_{5}$ so we can suppose $c=c_{1}, c_{2}=c_{3}$. After multiplying the third congruence by $c^{-1}$ and taking suitable linear combinations of $f, g, h$ the coefficient matrix becomes

$$
\left[\begin{array}{cccccccc}
1 & 0 & 0 & a_{1} & a_{2} & a_{3} & a_{4} & a_{5} \\
0 & 1 & 0 & b_{1} & b_{2} & b_{3} & b_{4} & b_{5} \\
0 & 0 & 1 & 1 & c & c & c_{4} & c_{5}
\end{array}\right]
$$

with $c, c_{4}, c_{5} \in S$. Further, since the columns are in general position, all the coefficients appearing are non-zero. We relabel so that $c_{4} \leq c_{5}$.

If $c_{4}=c_{5}$ then $x, y,-u, u, v,-v, w,-w$ satisfies the third congruence and puts the first 2 congruences into the form

$$
\begin{aligned}
x^{3}+a_{1} u^{3}+\left(a_{2}-a_{3}\right) v^{3}+\left(a_{4}-a_{5}\right) w^{3} & \equiv 0(\bmod p), \\
y^{3}+b_{1} u^{3}+\left(b_{2}-b_{3}\right) v^{3}+\left(b_{4}-b_{5}\right) w^{3} & \equiv 0(\bmod p) .
\end{aligned}
$$

If " $q^{*} \geq 3$ " then these congruences have a solution of rank 2 , using Lemma 20. If " $q^{*}<3$ " then 3 of the columns lie in a 1-dimensional space and we have a non-trivial solution of a single ternary cubic $\alpha \zeta^{3}+\beta \xi^{3}+\gamma \eta^{3} \equiv 0$. This leads to a non-trivial solution of the system of 3 congruences, and since $q=6$ the solution will be non-singular.

We are now left with 9 forms $h_{0}$ with coefficients

$$
\begin{array}{llllllll}
0 & 0 & 1 & 1 & 1 & 1 & 1 & A \\
0 & 0 & 1 & 1 & 1 & 1 & 1 & B \\
0 & 0 & 1 & 1 & 1 & 1 & A & B \\
0 & 0 & 1 & 1 & A & A & 1 & A \\
0 & 0 & 1 & 1 & A & A & 1 & B \\
0 & 0 & 1 & 1 & A & A & A & B \\
0 & 0 & 1 & 1 & B & B & 1 & A \\
0 & 0 & 1 & 1 & B & B & 1 & B \\
0 & 0 & 1 & 1 & B & B & A & B .
\end{array}
$$


For each of these forms we would form a list of all non-trivial solutions of $h_{0} \equiv 0(\bmod p)$. The computer then runs through all the $(p-1)^{10}$ possible forms $f_{0}, g_{0}$ and checks that there is a common solution, or lists $f_{0}, g_{0}, h_{0}$ as a counter-example.

14. The case $q=5$, computations. Here $p>13$ and we can write the coefficient matrix in the form

$$
\left[\begin{array}{cccccccc}
1 & 0 & a & 0 & a_{1} & a_{2} & a_{3} & a_{4} \\
0 & 1 & b & 0 & b_{1} & b_{2} & b_{3} & b_{4} \\
0 & 0 & 0 & 1 & 1 & c_{2} & c_{3} & c_{4}
\end{array}\right]
$$

where $c_{2}, c_{3}, c_{4}$ and $b \in S=\{1, A, B\}$, either $c_{2}=c_{3}$ or $\left\{c_{2}, c_{3}, c_{4}\right\}=$ $\{1, A, B\}$. The argument of $\S 10$ fails only when $a$ and $b$ are cubic residues, so now $b=1$ and $a$ is a cubic residue $(\bmod p)$.

Further, the argument of $\S 10$ fails only when the congruences

$$
\begin{aligned}
x^{3}+a w^{3}+a_{1} \zeta^{3} & \equiv 0, \\
y^{3}+w^{3}+b_{1} \zeta^{3} & \equiv 0
\end{aligned}
$$

have no solution with $\zeta \not \equiv 0$. We begin by forming a list $L$ of the triples of coefficients $a, a_{1}, b_{1}$ for which this can happen, with $a$ a cubic residue.

There are now 10 possible forms $h_{0}$, where $c_{2}, c_{3}, c_{4}$ are

$\begin{array}{lll}1 & 1 & c \\ A & A & c \\ B & B & c \\ 1 & A & B\end{array}$

with $c \in S$, after reordering the variables. For each $h_{0}$ we could form a list of the non-trivial solutions to $h_{0} \equiv 0(\bmod p)$ and then run through all possible $f_{0}, g_{0}$ by letting $a, a_{1}, b_{1}$ run through the values in the list $L$, and letting $a_{2}, a_{3}, a_{4}, b_{2}, b_{3}, b_{4}$ run through all $p^{6}$ possible values. For each pair $f_{0}, g_{0}$ we run down the list of solutions to $h_{0} \equiv 0$ to see if there is a common solution of rank $3(\bmod p)$. If there is not then we check the coefficients to see that $r=1$ and $q=5$. If these conditions are satisfied we list the triple $f_{0}, g_{0}, h_{0}$ as a counter-example.

15. The case $q=4$. In this case we only consider $p=19$ and the coefficient matrix is

$$
\left[\begin{array}{cccccccc}
1 & 0 & \alpha_{1} & \alpha_{2} & 0 & a_{1} & a_{2} & a_{3} \\
0 & 1 & \beta_{1} & \beta_{2} & 0 & b_{1} & b_{2} & b_{3} \\
0 & 0 & 0 & 0 & 1 & 1 & c_{2} & c_{3}
\end{array}\right]
$$

where $c_{2}, c_{3}, \beta_{1}, \beta_{2} \in S$. On ordering the variables so that $c_{2} \leq c_{3}$ there are 6 forms $h_{0}$ to consider and for each $h_{0}$ we begin by forming a list of all nontrivial solutions to $h_{0} \equiv 0(\bmod p)$. 
The argument of $\S 11$ fails only if the congruences (2.63) have no solution with $\zeta \not \equiv 0$. We run through all possible coefficients, with $\beta_{i} \in S, \alpha_{1} \beta_{2}-$ $\alpha_{2} \beta_{1} \not \equiv 0$ and form a list $L^{\prime}$ of the coefficients $\beta_{1}, \beta_{2}, \alpha_{1}, \alpha_{2}, a_{1}, b_{1}$ for which there is no solution with $\zeta \not \equiv 0(\bmod p)$.

For each of the 6 forms $h_{0}$ we run through all possible pairs of forms $f_{0}, g_{0}$ by letting $a_{2}, a_{3}, b_{2}, b_{3}$ run through all possible values, and the other coefficients run through the list $L^{\prime}$. For each pair $f_{0}, g_{0}$ we run through all the solutions of $h_{0} \equiv 0(\bmod p)$ to see if there is a common solution of rank $3(\bmod p)$. If there is not then we check the coefficients to see that $r=1$ and $q=4$. If these conditions are satisfied we list the triple $f_{0}, g_{0}, h_{0}$ as a counter-example.

\section{References}

[1] M. Aigner, Combinatorial Theory, Springer, Berlin 1979.

[2] R. C. Baker, Diagonal cubic equations II, Acta Arith. 53 (1989), 217-250.

[3] R. C. Baker and J. Brüdern, On pairs of additive cubic equations, J. Reine Angew. Math. 391 (1988), 157-180.

[4] B. J. Birch, Homogeneous forms of odd degree in a large number of variables, Mathematika 4 (1957), 102-105.

[5] R. Brauer, A note on systems of homogeneous algebraic equations, Bull. Amer. Math. Soc. 51 (1945), 749-755.

[6] J. Brüdern, On pairs of diagonal cubic forms, Proc. London Math. Soc. (3) 61 (1990), 273-343.

[7] J. Brüdern and R. J. Cook, On pairs of cubic diophantine inequalities, Mathematika, to appear.

[8] N. G. de Bruijn, The asymptotic behaviour of a function occurring in the theory of primes, J. Indian Math. Soc. (N.S.) 15 (1951), 25-32.

[9] S. Chowla, H. B. Mann and E. G. Straus, Some applications of the CauchyDavenport theorem, Kon. Norske Vidensk. Selsk. Forh. 32 (1959), 74-80.

[10] R. J. Cook, A note on a lemma of Hua, Quart. J. Math. Oxford Ser. 23 (1972), $287-288$

[11] —, Pairs of additive equations, Michigan Math. J. 19 (1972), 325-331.

[12] - Pairs of additive congruences: cubic congruences, Mathematika 32 (1985), 286300.

[13] —, Simultaneous additive congruences, Kon. Norske Vidensk. Selsk. Skr. 5 (1985), $1-7$.

[14] H. Davenport and D. J. Lewis, Homogeneous additive equations, Proc. Roy. Soc. London A274 (1963), 443-460.

[15] - - - Cubic equations of additive type, Philos. Trans. Roy. Soc. London A261 (1966), 97-136.

[16] - - - Two additive equations, in: Proc. Sympos. Pure Math. 12, Amer. Math. Soc., 1967, 74-98.

[17] - - - Simultaneous equations of additive type, Philos. Trans. Roy. Soc. London A264 (1969), 557-595.

[18] M. M. Dodson, Homogeneous additive congruences, ibid. A261 (1966), 163-210. 
[19] D. J. Lewis, Cubic congruences, Michigan Math. J. 4 (1957), 85-95.

[20] L. Low, J. Pitman and A. Wolff, Simultaneous additive congruences, J. Number Theory 29 (1988), 31-59.

[21] E. Stevenson, The Artin conjecture for three diagonal cubic forms, ibid. 14 (1982), 374-390.

[22] R. C. Vaughan, On pairs of additive cubic equations, Proc. London Math. Soc. (3) 34 (1977), 354-364.

[23] - The Hardy-Littlewood Method, University Press, Cambridge 1981.

[24] —, On Waring's problem for cubes, J. Reine Angew. Math. 365 (1986), 122-170.

[25] - A new iterative method in Waring's problem, Acta Math. 162 (1989), 1-71.

DEPARTMENT OF PURE MATHEMATICS

UNIVERSITY OF SHEFFIELD

HICKS BUILDING, HOUNSFIELD ROAD

SHEFFIELD S3 7RH, U.K.
MATHEMATISCHES INSTITUT

BUNSENSTRASSE 3-5 3400 GÖTTINGEN

FEDERAL REPUBLIC OF GERMANY 